\title{
On the youthful writings of Louis J. Mordell on the Diophantine Equation $y^{2}-k=x^{3}$
}

\author{
Sébastien Gauthier* \& François Lề \\ Postprint version. April 2019.
}

\begin{abstract}
This article examines the research of Louis J. Mordell on the Diophantine equation $y^{2}-k=x^{3}$ as it appeared in one of his first papers, published in 1914. After presenting a number of elements relating to Mordell's mathematical youth and his (problematic) writing, we analyze the 1914 paper by following the three approaches he developed therein, respectively based on the quadratic reciprocity law, on ideal numbers, and on binary cubic forms. This analysis allows us to describe many of the difficulties in reading and understanding Mordell's proofs, difficulties which we make explicit and comment on in depth.
\end{abstract}

\section{Introduction}

When he died in 1972, Louis Joel Mordell had accumulated numerous mathematical honours. ${ }^{1}$ He had been Sadleirian Professor of pure mathematics in Cambridge between 1945 and 1953, and President of the London Mathematical Society between 1943 and 1945; he had been elected a Fellow of the Royal Society in 1924, and was foreign member of the Academies of Oslo, Uppsala, and Bologna; the list of the institutions from all around the world where he had been asked to be a visiting speaker ran to almost 200 items; he had been awarded the De Morgan Medal in 1941, the Senior Berwick Prize in 1946, and the Sylvester Medal in 1949 "for his distinguished researches in pure mathematics, especially for his discoveries in the theory of numbers"; ${ }^{2}$ he was recognized for having built "a strong school of mathematics in Manchester" during the 1930s; he was famous for the "finite basis theorem" which he had proved in a paper of 1922; there was a "Mordell-Weil theorem" and a "Mordell conjecture";

\footnotetext{
*Univ Lyon, Université Claude Bernard Lyon 1, CNRS UMR 5208, Institut Camille Jordan, 43 blvd. du 11 novembre 1918, F-69622 Villeurbanne Cedex, France.

†Univ Lyon, Université Claude Bernard Lyon 1, CNRS UMR 5208, Institut Camille Jordan, 43 blvd. du 11 novembre 1918, F-69622 Villeurbanne Cedex, France.

${ }^{1}$ The information of this paragraph comes from the obituary of Mordell written by his former student John Cassels, [Cassels 1973]. See also [Davenport 1964; Cassels 1986].

${ }^{2}$ About the Sylvester Medal, see [Grattan-Guinness 1993], and particularly p. 108 for the quote on Mordell.

${ }^{3}$ In modern terms, the finite basis theorem (sometimes also called "Mordell's theorem") states that the group of rational points on an elliptic curve defined over the field of rational numbers is finitely generated. The Mordell-Weil theorem generalizes the latter to the case of an Abelian variety defined over a number field. The Mordell conjecture, now Falting's theorem, states that the group of rational points on a curve of genus greater than 1 and defined over the rationals is finite.
} 
finally, there was a "Mordell equation," namely the Diophantine equation $y^{2}-k=x^{3}$.

This litany of distinctions, however, contrasts with the small number of historical investigations that have been devoted to Mordell. Indeed, apart from some papers where his name appears once or twice-mostly for the 1922 finite basis theorem-very few other texts have given more room to his works. ${ }^{4}$ Thus the proof of the finite basis theorem has been analyzed in the specific perspective of a historical study of the method of infinite descent, [Goldstein 1993], and, more recently, the research of Mordell (among others) on the geometry of numbers between 1923 and 1945 has been scrutinized, both to delineate the way geometry intervened in this research and to discuss the relevance of the notions of "discipline" and "school" to describe the activities linked with it, [Gauthier 2007, 2009].

Our aim in this paper is to analyze another facet of Mordell's research, the facet related to the Diophantine equation $y^{2}-k=x^{3}$. As Mordell himself explained in his Reminiscences of an Octogenarian Mathematician, this equation "played a prominent part in [his] research" throughout his entire career [Mordell 1971, pp. 956-957] — significantly, it had been the topic of his 1945 inaugural lecture as Sadlerian Professor. ${ }^{5}$ This equation, considered as an equation with integral unknowns $x, y$ and parameter $k$, had been the object of his first substantial published paper, dated 1912 and published in 1914, [Mordell 1914a] ${ }^{6}$ for which he was awarded the second Smith's Prize of $1912,{ }^{7}$ and which contains results about the possibility of solving the equation depending on the values of $k$. Mordell came back on the equation $y^{2}-k=x^{3}$ a few years later, in 1920, proving that it has at most a finite number of integral solutions for any integral parameter $k$, [Mordell 1920]. As for the problem of determining the rational solutions, this was tackled decades later, in papers published in 1947, 1966, and 1968, [Mordell 1947b, 1966, 1968]. ${ }^{8}$

Here we will concentrate on the research of Mordell on the equation $y^{2}-k=x^{3}$ when considered as an equation between integers, specifically on the 1914 paper dealing with this equation. Our aim being to give a precise view of Mordell's practice in this paper, we will first present a short account of his mathematical education, paying special care to what relates to number theory. After a few words on his problematic mathematical writing, we will

\footnotetext{
${ }^{4}$ See for instance [Schappacher and Schoof 1996; Roquette 1998; Houzel 2004]. Note that [Schappacher 1991] devoted a few lines to Mordell's theorem, and explained that this theorem was originally not expressed in group-theoretic terms. Mordell also appears in a few papers which are not dedicated to the history of number theory: see for example [Fletcher 1986] about the reaction of Britain to the explusion of mathematicians of the Nazi Germany, [Barrow-Green 1999] about the history of the Smith Prize, or [Rice and R. J. Wilson 2003] about the development of analysis in Britain in the twentieth century. Finally, Mordell's time in London (1913-1920) has been lately described in [Fairbairn 2017].

${ }^{5}$ The lecture has been published two years later under the title A Chapter in the Theory of Numbers [Mordell 1947a]. That Mordell came back on the equation $y^{2}-k=x^{3}$ again and again has also been remarked in [Cassels 1973, p. 496] for instance.

${ }^{6}$ This paper is the second item on the list of Mordell's publications, [Cassels 1973, pp. 511-520]. As will be seen, the first item [Mordell 1913] is a short note which is actually an outgrowth of the research that led to the 1914 paper.

${ }^{7}$ The Smith's Prize competition was intended for postgraduates students in Cambridge, and consisted in submitting an essay supposed to display the ability of the candidate to produce an original work. At the beginning of the twentieth century, the PhD did not exist in Great Britain and, as June Barrow-Green explains, "the Smith's Prize competition provides one of the few explicit sources of information about Cambridge postgraduate research in mathematics." [Barrow-Green 1999, p. 304].

${ }^{8}$ As of today, the question of determining the parameters $k$ for which the equation has a finite number of rational solutions is still open; it is linked to the Birch and Swinnerton-Dyer conjecture.
} 
analyze the paper on $y^{2}-k=x^{3}$. The analysis will be split into three sections corresponding to the three mathematically independent points of views that Mordell adopted: the first one uses congruences and the quadratic reciprocity law, the second one is based on the notion of "ideal numbers," and the third one bears on the theory of binary cubic forms. We will enter the technique in depth, both to present in fine detail Mordell's mathematical practice and to highlight a number of issues linked to the very writing of the paper, and which arise when one tries to read it closely. Focusing on these issues will produce a certain image of this paper, an image which we will comment on in our concluding remarks.

\section{A self-educated number theorist in Cambridge}

\subsection{Toward the Mathematical Tripos}

If Mordell may nowadays mostly be known as an English mathematician, he was actually born in Philadelphia in the United States on January 28, 1888, and he moved in Cambridge at the age of almost 20, in 1907-he took English citizenship more than twenty years later, in December 1929. ${ }^{9}$ He seems to have had an education that was standard for an American boy of the time, as he successively attended primary school (from the age 6 to 10), grammar school (10 to 14), and high school (14 to 18 ).

In his autobiographical notes, Mordell remembered having discovered his taste for algebra just before entering high school, at the age of 13 or 14, when he found old books on this subject in a bookstore. The two books that Mordell specified are Charles Hackley's Treatise of Algebra in its third edition of 1849, and Joseph Ficklin's Complete Algebra, published in 1875, [Hackley 1849; Ficklin 1875]. Interestingly, Mordell retrospectively placed the seeds of his arithmetical predilections in the book of Hackley: ${ }^{10}$

It was a really good book, though not rigorous, and contained a great deal of material including the theory of equations, series, and a chapter on the theory of numbers. It, like the old algebras of these days, had a chapter on Diophantine analysis, a subject I found most attractive. It is not without interest that in later years much of my best research deals with this. [Mordell 1971, pp. 953-954]

Hackley's chapters on the theory of equations comprised various sections bearing on topics such as equations of degree 1 to 4 , the approximation of roots, binomial equations, elimination, etc. The chapter on number theory pertained to the notion of divisibility, expounded the theory of congruences, and ended up with Fermat's little theorem and the possible residues of square numbers modulo 4 and 5. As for the chapter on Diophantine analysis, it presented a list of problems and solutions in the style of Diophantus' Arithmetica: for instance, while the first problem was "[t]o find two square numbers whose sum is a square," the last one was "to find three integral square numbers, such, that the difference of every two of them shall be a square number" [Hackley 1849, pp. 458, 465].

During the following years, when he was in high school, Mordell studied other mathematical textbooks, and he noticed that many of the problems that were posed therein were

${ }^{9}$ The biographical elements of this subsection come from the autobiographical writings [Mordell 1959, 1971]. Note that these writings have served to a great extent as a base for later papers on Mordell's life, such as [Davenport 1964; Cassels 1973, 1974; Fairbairn 2017].

${ }^{10}$ Mordell did not present Ficklin's book with the same level of precision. 


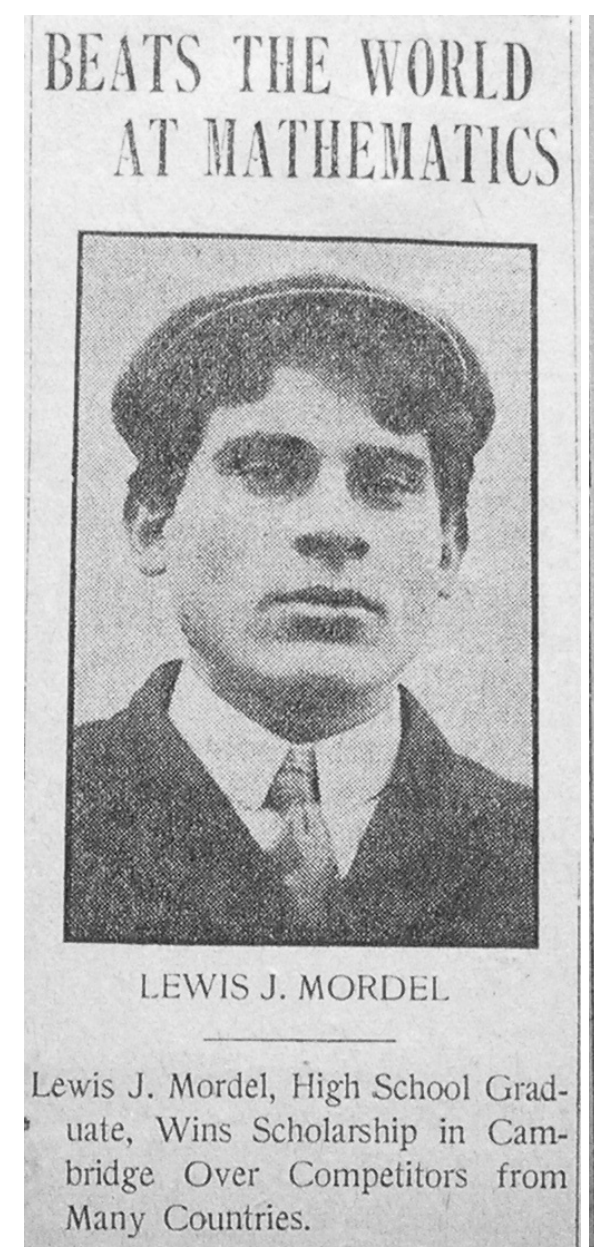

Lewis J. Mordel, a graduate of the Central High School, brought additional honors to his alma mater yesterday, when he was awarded a three-year scholarship in mathematics by St. John's College, Cambridge, England.

Mordel went to Cambridge with nothing but his High School training and competed against graduates of schools and colleges in every part of the world. The examinations were open to all com petitors, but for the first time a High School graduate was entered against college men. His entry created laughter instead of serious consideration, but at the conclusion of the examinations, which lasted four days, he stood No. 1 of 250 applicants, with an average of a trifle below 100 .

At the Central High School Mordel's ability along mathematical lines was regarded by the members of the faculty as phenomenal. In his Sophomore year he had completed the mathematical course provided for the four-year course and during his last two years in the sehool he took up the higher mathematies.

Tro support himself he devoted seven hours of every day to cosohing his tellow-students, and on one occasion bis. and on one occaslont stood at a blackboard for forty-eight hours in an endeavor to pull a student through an examination. And the examination was passed. At the end of his Senior year he devoted all his time to coaching, having no examinations to take, and in this manner earned enough money to toke him to England.

Mordel's present aim is to cover his Mordel's present aim is to cover his three years' work sufficiently well to entitle him to a fellowship for four ad-
ditional years.

Figure 1 - Press cutting from The Philadelphia Press (January 10, 1907) conserved in St John's College Library, Papers of Louis Joel Mordell (box 4, folder 41). By permission of the Master and Fellows of St John's College, Cambridge.

taken from Cambridge scholarship examinations and from the Mathematical Tripos. ${ }^{11}$ This, he explained, triggered his desire to sit the examination to be admitted to one of the colleges of Cambridge, where he could study mathematics. Mordell did so in December 1906 and was ranked first - this success created a little local fame in Philadelphia, as a press cutting from The Philadelphia Press dated January 10, 1907 attests (see figure 1).

The studies in Cambridge began in October 1907 at St John's College, with the support of both a scholarship from this college and another one from Mordell's former high school in Philadelphia. We do not know for sure which lectures he attended at St John's, but in his Reminiscences he evoked the names of Robert Rumsey Webb, "who lectured in applied mathematics," of Thomas John l'Anson Bromwich, and of Henry Frederick Baker, who was

\footnotetext{
${ }^{11}$ We recall that the Tripos is a competitive exam that was taken by second-year or third-year students of colleges of Cambridge, which thus marked the end of the undergraduate studies. It led to a ranking of students, of which the first ones were called the Wranglers. The ranking was abandoned in 1909, after a reform of the exam. See [D. B. Wilson 1982; Warwick 2003; Crilly 2011].
} 
the director of studies and his personal supervisor, a supervisor described as "perhaps distant, or unsympathetic." [Mordell 1971, pp. 955-956]. Mordell added that Baker had tried, in vain, to steer him towards geometry, making him attend the lectures of Herbert William Richmond on higher plane curves, and of Andrew Forsyth on differential geometry. Yet he rapidly dropped them, and, more generally, he only attended a few lectures. Retrospectively, Mordell considered that Godfrey Harold Hardy would have been a better supervisor than Baker:

\begin{abstract}
[Hardy] would have known how to deal with a self-educated mathematician, who rather unwisely did not appreciate the advantages of lectures. He would have taught me worldly wisdom and the ways of the world as well as mathematics from a modern point of view. This would have been very important as the older books which I had first come across were lacking in rigor. [Mordell 1971, p. 956]
\end{abstract}

We note that the preference for Hardy (expressed a posteriori) is associated with the lack of rigour resulting from Mordell's early mathematical readings and self-education, and echoes the usual story according to which Hardy was the mathematician who brought rigour, and especially analysis, into British mathematics at the beginning of the twentieth century. ${ }^{12}$ As announced in our introduction, the 1914 paper of Mordell on the equation $y^{2}-k=x^{3}$ contains a number of writing issues, which may be linked to the lack of rigor alluded to in the previous quotation.

Mordell prepared for the Mathematical Tripos from his entrance at St John's in October 1907 until 1909, when he took the exam and was ranked third Wrangler. As was usual for Wranglers who wished to continue with mathematics, he then prepared the so-called Part III of the Tripos. It is at this time (around 1910-1911) that Mordell started his research in number theory that would lead to the paper we are interested in.

\title{
1.2 The theory of numbers in Great Britain: Mordell's view
}

Mordell reported a lack of interest for number theory in Great Britain in the beginning of the twentieth century at two occasions at least. Already in an essay published in 1918 in Science Progress, after having named Henry John Stephen Smith and James Joseph Sylvester as the two only significant English arithmeticians of the nineteenth century, he proposed several reasons for why number theory was not in favour in the country before World War I, [Mordell 1918]. ${ }^{13}$ First and foremost, he emphasized that English mathematicians of the time tended to neglect such a subject because its "conclusions did not have any practical importance." $\mathrm{He}$ also criticized the educational system because of the excessive focus that was put on the examinations themselves, and hence on the courses that served to cover the topics which were potential matter for these examinations: this tended to obscure students from some facets of pure mathematics that were not included in the exam syllabus, and to favour the production of basic textbooks to the detriment of more advanced treatises. Finally, Mordell asserted that

\footnotetext{
${ }^{12}$ See [Rice and R. J. Wilson 2003]. Hardy was a lecturer in Cambridge in these years, but not at St John's: he entered Trinity College as a student in 1896, and was a lecturer there between 1906 and 1919, [Titchmarsch 1949, p. 448].

${ }^{13}$ Catherine Goldstein [1999, p. 396] remarked that between 1870 and 1914, a great part of the English number-theoretical production consisted in elementary papers published in Educational Times, which may explain Mordell's point of view if the latter aimed at number theory as published in research journals. However, because of the rather small number of historical investigations about number theory in Great Britain at the end of the nineteenth century, it is difficult for us to assess more precisely Mordell's statements.
} 
the Fellowship system forced young scholars to produce mathematical papers rapidly, which would discourage them from exploring topics that required a great amount of time to enter. This, he continued, was the case for number theory, in particular because almost no advanced lectures on this topic existed in Great Britain at the beginning of the century.

Much later, in the Reminiscences of an Octogenarian Mathematician, Mordell repeated that "[n]umber theory was little studied in Britain in [the beginning of the twentieth century]. There were only Professor G. B. Mathews at Bangor, who wrote a book on number theory, and J. H. Grace of Peterhouse, who wrote a few papers on the subject." [Mordell 1971, p. 957]. ${ }^{14}$

John Hilton Grace had been a lecturer at Peterhouse Cambridge since 1897. Among the 37 publications of his that are reviewed in Jahrbuch über die Fortschritte der Mathematik, 22 are classified as geometry and 10 are in the section devoted to the algebraic theory of forms, ${ }^{15}$ while only 5 (all published in 1917 or in 1919) belong to number-theoretic sections: 4 of them deal with rational approximation, and the remaining one is about the decomposition of integers as sums of four squares. These are probably the papers referred to by Mordell in 1971, but their publication dates prove that they could not have served as references for the preparation of the 1914 paper on the equation $y^{2}-k=x^{3}$. That being said, as a student Mordell could have known Grace as a lecturer in a neighbouring college in Cambridge. Moreover, as we know that there were lectures in (probably elementary) number theory in Cambridge at least until 1907 [D. B. Wilson 1982, App. 1], and that Grace gave such lectures at least in 1913, 1919, and 1921 [Barrow-Green 2014, pp. 66-67, 115], it is possible that he lectured on this subject during Mordell's stay in Cambridge as well. Grace also lectured in invariant theory at that time, a theory which Mordell used some elements of in his works on the equation $y^{2}-k=x^{3}$. Thus he may have been in contact with it through these lectures or through Grace's writings on the subject.

The case of George Ballard Mathews is different from that of Grace, for he was not in Cambridge but in Bangor (Wales) when Mordell was a student. The book that Mordell refers to is certainly Theory of Numbers, published in 1892, [Mathews 1892]. Since Mordell cited this book in one of his first papers [Mordell 1915], it is likely that he had become acquainted with it when he was a student in Cambridge. As Mathews wrote, his Theory of Numbers was intended to "give a fairly complete account of the theories of congruences and of arithmetical forms" [Mathews 1892, p. v]. Accordingly, two chapters deal with congruences and culminate in a proof of the law of quadratic reciprocity, six chapters develop the theory of binary quadratic forms, and the two last chapters are respectively devoted to cyclotomy and to the distribution of prime numbers. It is interesting to note that congruences, the law of quadratic reciprocity, and the theory of quadratic forms are part of the knowledge that Mordell would use in the paper about the equation $y^{2}-k=x^{3}$. Other objects, which are not to be found in Mathews' book, such as binary cubic forms and ideal numbers, were

\footnotetext{
${ }^{14}$ Curiously, Mordell did not mention the name of James Whitbread Lee Glaisher, who was still active in number theory at the beginning of the twentieth century. Further, remark that Hardy and John Edensor Littlewood were mostly concerned with questions of analysis at that time.

${ }^{15}$ These ten references about the algebraic theory of forms include a 1903 book written with Alfred Young, entitled The Algebra of Invariants, "which first presented the work of Clebsch and Gordan to British mathematicians." [Todd 1958, p. 94]. Note that the great number of geometric publications in comparison with the others echoes the usual presentation of Grace as a geometer, [Todd 1958, p. 94], [Barrow-Green and Gray 2006, p. 328 sqq.].
} 
also used in this paper: strikingly, they are the topics of several papers that Mathews had published at the turn of the century, such as [Mathews 1891, 1893a,b, 1911]. We have no direct evidence that Mordell knew these papers in the early 1910s, but their existence allows us to see that the various objects that would be used by Mordell for the equation $y^{2}-k=x^{3}$ were part and parcel of Mathews' works. ${ }^{16}$

The preceding paragraphs show that even if Mordell repeatedly presented himself as a self-educated mathematician, he was not a mathematician with no resources, in particular in number theory. Apart from Grace's lectures and the book (and papers) by Mathews that we just mentioned, our analysis of his 1914 paper will show that Mordell had a good knowledge of the works of many authors from the nineteenth century. ${ }^{17}$ The quotation about Hardy that we gave in the previous subsection indicates that Mordell linked his self-education not so much to an ignorance of past and current arithmetic research, but rather to the lack of a mentor who would have shared with him a certain global vision of the world and prepared his entrance in the research universe, in particular by teaching him the appropriate codes. This issue is particularly true when reading the published research of the young Mordell.

\subsection{On Mordell's early writing}

In his biographical essay on Mordell, Cassels [1973] devoted some lines to a paper of Mordell which is different from the one we are mainly interested in here, but which was published the same year and also dealt with Diophantine equations, [Mordell 1914b]. Mordell had submitted this paper when he was proposed for a Fellowship at St John's, which application failed, and it had been refused by the London Mathematical Society as well. Trying to give reasons for these rejections, Cassels emphasised that "like nearly all of Mordell's early papers it is written in a narrative style without the conventional enunciation of theorems etc. and it is difficult to disentangle what it is that is actually proved." [Cassels 1973, p. 498]. He then suggested that these problems were in part explained by Mordell's mathematical self-education: "He would clearly have benefited greatly from advice on how actually to present a piece of mathematics," a statement which echoes the descriptions that Mordell made of himself and that we reported above.

Cassels' remarks, of course, must be read cautiously, for neither was he an historian of mathematics, nor was he born at the time of the publications he commented upon: Cassels was born in 1922, that is, almost 10 years after these publications, so that his impressions about an unconventional style may reflect a difference of mathematical education and an anachronistic view of Mordell's early writings. Now, it is true that, in the paper [Mordell 1914b], Mordell did not delimit his results and demonstrations by introducing labels such as "Theorem" and "Proof," or by using italics for the statement of the theorems. Instead, while some of them are preceded by sentences such as: "We have the following results," others are simply written in the flow of the text. Moreover, Mordell was not the only one to do so in 1914: among the 27 papers that appeared in the same volume of Proceedings of the

\footnotetext{
${ }^{16}$ Nevertheless, we will see and discuss the fact that Mordell used "ideal numbers" whereas Mathews (among others) dealt with "ideals."

${ }^{17}$ Let us add that Henry John Stephen Smith's Report on the Theory of Numbers is among the recurring references cited by Mordell in his other papers published in the 1910s. Just as with Mathews' book, Mordell thus could have known this Report when he was studying in Cambridge.
} 
London Mathematical Society, about the third did not any use specific means to emphasize their results. ${ }^{18}$

The comments formulated by Cassels must therefore be qualified, but they still testify of serious issues that may arise when trying to read and understand the technique of the paper [Mordell 1914b], even for a confirmed mathematician such as Cassels. Let us additionally remark that other features are likely to make such a reading complicated: hypotheses are scattered through the paper, letters appearing in a proof are not well defined, the very formulation of some sentences makes their status (as new hypothesis or result to be proved, for instance) unclear, etc. As we will see in the next sections, similar issues can be observed in the case of the 1914 paper on the equation $y^{2}-k=x^{3}$.

In fact, Mordell's way of writing had also been seen as problematic by mathematicians who were his contemporaries: the archives of Mordell that are conserved at St John's include reports that had been written by anonymous referees of some of his papers of the 1920s, and of which a great number contain serious criticism. For instance, a report on a paper which had been submitted around 1929 to the London Mathematical Society emphasises that:

It is a pity that the paper should be so marred by obscurities of style and misprints which are so numerous. [...] Evidently the paper should be printed: but very drastic reconsideration of style and type and manner of notation and arrangement should be required, from such an experienced author. ${ }^{19}$

As we said, similar opinions of referees appear recurrently in Mordell's archives; they all insist on the "obscurities of style" of the papers of Mordell, obscurities that are be linked to the overall presentation of the papers, to the notations employed therein, or even to the grammar and the punctuation used by Mordell.

Mordell's response to the criticism that we quoted above attests that he was used to such bad evaluations, and the counterarguments he uses certainly do not pertain only to the one paper in question there. ${ }^{20}$ First, Mordell asserted that it was normal that his papers were difficult to read because mathematics itself is highly demanding. For him, the bad appraisal of his "obscure style" only showed the lack of understanding of his readers, and making things easier for them was not his duty. These arguments-which display a degree of bad faith and condescension - were combined with a more general critique of one facet of the referee system, the facet which "sometimes tend to suppress the personality and individuality of the writer."

Reflecting all these testimonies, our own analysis of Mordell's paper on $y^{2}-k=x^{3}$ confronted us with a number of issues that make its reading and understanding arduous:

\footnotetext{
${ }^{18} \mathrm{On}$ the contrary, all the theorems and the proofs in the two papers of Hardy published in this volume are clearly delimited, by the use of italics and of labels "Theorem" and "Proof." More generally, we are not suggesting that the issues about Mordell's writing that we will describe throughout our paper are either exclusively proper to him or completely widespread in the mathematical literature of the beginning of the twentieth century. Our aim here is to focus on Mordell's way of writing and its consequences in the context of the paper on $y^{2}-k=x^{3}$, and not to evaluate to what extent such issues could be common at the beginning of the twentieth century, which would lead us far beyond the limits of our paper.

${ }^{19}$ Letter of F. P. White (Honorary Secretary of the London Mathematical Society) to Mordell, dated June 18, 1929, and reporting the comments of an anonymous referee. The letter is kept in Mordell's papers in the archives of St John's College (box 2, folder 15).

${ }^{20}$ The letter containing this response is addressed to the Council of the London Mathematical Society. It is kept in Mordell's papers in the archives of St John's College (box 6). Note that these archives contain almost no material from the 1910s.
} 
notations are far from stable (even from one line to the other); technical passages which require further justifications or fastidious computations are left unexplained and are not signalled as problematic; sufficient conditions which have been brought to light in the middle of a proof surreptitiously become hypotheses for the rest of the paper; problems with the logical structure or with the formulation of sentences make some proofs and results questionable; the status of propositions appearing in the middle of a proof is not clear-are they new hypotheses, knowledge that is supposed to be common, or properties which must be proved?-, hypotheses that are made at some point are not recalled when Mordell seems to sum up his results; such summaries are neither systematically self-contained, nor clearly distinguished from the rest of the paper, etc.

We think that all these issues, being part and parcel of Mordell's early mathematical practice, should be included in a historical study aiming at the description of this practice and, in this respect, we wish to share them faithfully with our own readers. ${ }^{21}$ Thus we searched for an adequate grain for our description of Mordell's mathematics, a grain sufficiently fine not to erase the details and particularities of Mordell, and at the same time not too fine, which would lead to an overly fastidious picture. We chose to present and analyze the technique in minute detail only for some specific parts of the paper, while the rest will be depicted less precisely. Moreover, in order to bring to light the issues as they present themselves in the paper, we will display extracts which, in our view, are representative of Mordell's way of writing. These choices will make many technicalities appear, but it is the analysis of these technicalities that will help us make our point about Mordell's paper.

Let us now delve into this paper. As stated above, Mordell divided it into three parts, each of them corresponding to a specific approach of the equation. The first one, which aimed at "find[ing] general formulæ for $k$, for which there are no solutions" [Mordell 1914a, p. 60], involved many fastidious computations and made a central use of the law of quadratic reciprocity. It was preceded by the introduction of the paper in which Mordell gave a number of references on the subject, references which mostly concerned the first part.

\section{Finding insoluble equations with the quadratic reciprocity law}

As was quite usual for number-theoretic papers of the time, Mordell started by summoning the name of Pierre Fermat, thus creating a lineage between his works on the equation $y^{2}-k=x^{3}$ and those of the French mathematician. "This equation," he reported, "was brought into prominence by Fermat, who had proposed as a problem to the English mathematicians, to shew that there was only one integral solution of the equation $y^{2}+2=x^{3}$ " [Mordell 1914a, p. 60]. Mordell quoted Fermat (in French, via Brassinne's Précis des æeuvres mathématiques), and he asserted that the method of the latter for proving that the only (positive) integral solution of $y^{2}+2=x^{3}$ is given by $x=3, y=5$ had never been published, and thus was "not known at present." 22

\footnotetext{
${ }^{21}$ Similar questions about Augustin Louis Cauchy's research on number theory are raised in [Boucard 2013, p. 363].

${ }^{22}$ In fact, in a 1659 letter to Carcavi (published only at the very end of the nineteenth century), Fermat explained that this problem, together with other "negative" ones, could be solved with the method of infinite descent [Goldstein 1993, pp. 27-28]. About Fermat's works in arithmetic, we refer to the research of C. Goldstein, for instance [Goldstein 1995, 2009]. Moreover, in the quotation reported by Mordell, Fermat mentioned that
} 
Mordell then cited the works of mathematicians of the nineteenth century who contributed to the topic as well: Victor-Amédée Lebesgue, Camille Gerono, Ernest De Jonquières, Savino Realis, and Théophile Pépin. Apart from the latter, these authors were cited for papers that had been published in Nouvelles annales de mathématiques, a journal which was mainly intended for students (and their teachers) taking courses in the French classes préparatoires or studying for the licence or the agrégation. ${ }^{23}$ The cited papers either focused on specific equations, such as $x^{3}+7=y^{2}$ [Lebesgue 1869] or $x^{3}=y^{2}+17$ [Gerono 1877], which were proved to be impossible to solve in integers, or searched for formulas for the parameter $k$ to make the corresponding equation insoluble: for instance, generalizing results that De Jonquières [1878a] had obtained, Realis [1883] proved that for any integers $a$ and $b$, the equation $x^{3}+b^{2}\left( \pm 8 b-3 a^{2}\right)=y^{2}$ has no integral solutions. Mordell did not comment on these results, but he asserted that they could be "considerably extended": as will be seen, his paper indeed contained many similar formulas and values for $k$ yielding an impossible equation.

At the very end of the introduction, Mordell finally cited two papers of Allan Cunningham [1905, 1908] published Mathematical Questions and Solutions from "The Educational Times," another "intermediate journal." ${ }^{24}$ These articles, which were answers to previously posed questions, were cited in relation with a certain "tentative method for solving" the equation $y^{2}-k=x^{3}$, a method that we will describe at the end of this section.

\subsection{First technicalities, first issues}

Instead of considering the equation $y^{2}-k=x^{3}$ as it appeared in the introduction of the paper, Mordell started from the more specific form $y^{2}-k l b^{2}=x^{3}-k^{3} a^{3}$, where the number $k$ thus had not the same meaning as previously. His point of view consisted in starting from initial conditions on the parameters $k, l, a$, and $b$, and then in alternating between the derivation of different identities and the addition of supplementary conditions on the parameters which would eventually lead to an absurdity. As Mordell's proof crucially involved a repeated use of the so-called Jacobi symbol, we begin by recalling some technical points about this symbol before entering into the proof itself.

We first recall that for any odd prime $p$ and any integer $a$, the Legendre symbol $(a / p)$ is defined as follows: if $p$ divides $a$, then $(a / p)=0$; if not, $(a / p)=1$ if $a$ is a quadratic residue modulo $p$, i.e. if there exists an integer $x$ such that $x^{2} \equiv a \bmod p$, and $(a / p)=-1$ in the opposite case. Further, being given an odd positive integer $N$ and an integer $a$, the Jacobi symbol $(a / N)$ is defined by the formula $(a / N)=\prod(a / p)^{\alpha}$, where $N=\prod p^{\alpha}$ is the prime factorization of $N$. It follows from this definition that $(a / N)=(b / N)$ if $a \equiv b \bmod N$, and

a method of Claude-Gaspard Bachet de Méziriac allowed to find an infinite number of rational solutions to $y^{2}+2=x^{3}$. Nowadays, the equation $y^{2}-k=x^{3}$ is sometimes referred to as the "Bachet equation" or the "Mordell-Bachet equation."

${ }^{23}$ About this journal, see [Rollet and Nabonnand 2013]. Jenny Boucard pointed out to us that the subject of cubic and biquadratic Diophantine equations was quite intensively studied in Nouvelles annales between the end of the 1860s and the middle of the 1880s, especially by the people cited by Mordell, [Boucard 2019]. A doctoral dissertation is currently being prepared on this very subject by Alain Larroche. As for the papers of Pépin cited by Mordell were published in Liouville's Journal de mathématiques pures et appliquées and in Annales de la Société scientifique de Bruxelles, [Pépin 1875, 1882].

${ }^{24}$ About the "questions and solutions" genre in British journals in the eighteenth and nineteenth centuries, see [Despeaux 2014]. 
that $(a b / N)=(a / N)(b / N)$ and $(a / M N)=(a / M)(a / N)$ for any (odd) integers $a, b, M$, and $N$. Another, important property of the Jacobi symbol is the (generalized) quadratic reciprocity law: for any coprime, odd integers $N$ and $M$, one has $\left(\frac{N}{M}\right)\left(\frac{M}{N}\right)=(-1)^{(N-1)(M-1) / 4}$.

Mordell's proof began with what he called a "few preliminary considerations" bearing on the divisors of integers of the form $x^{2}-k y^{2}$. Specifically, Mordell showed that any odd prime divisor $q$ of $x^{2}-k y^{2}$ such that $(k / q)=-1$ is necessarily a common divisor of $x$ and $y .{ }^{25} \mathrm{He}$ then extended the result to the odd divisors of $x^{2}-k y^{2}$ : in Mordell's formulation, no odd number $t$ such that $(k / t)=-1$ can divide $x^{2}-k y^{2}$ unless all the prime factors $q$ of $t$ such that $(k / q)=-1$ divide both $x$ and $y$.

After these preliminary considerations, Mordell considered the equation $y^{2}-k l b^{2}=$ $x^{3}-k^{3} a^{3}$, where the parameter $k$ has no square factor and is prime to $b l$. Mordell did not explicitly explain why he privileged this form of equation, but, as the right-hand side is a difference of two cubes, he could factorize it at once:

$$
\begin{aligned}
y^{2}-k l b^{2} & =x^{3}-k^{3} a^{3} \\
& =(x-k a) N,
\end{aligned}
$$

with $N=x^{2}+k a x+k^{2} a^{2}$. Interestingly, several of the authors whom Mordell cited in his introduction had used similar manipulations. For instance, Gerono [1877], who studied the equation $y^{2}+17=x^{3}$, began by rewriting it in the form $y^{2}+5^{2}=x^{3}+2^{3}$, then factorized the right-hand side, and discussed the (im)possibility of the factors so obtained to be divisors of a sum of two squares, here $y^{2}+5^{2}$. Similarly, when Pépin [1882] tackled the equation $x^{3}+a=y^{2}$ with $a=c^{3}-4^{\alpha} b^{2}$, he transformed the equation into $y^{2}+\left(2^{\alpha}\right)^{2}=x^{3}+c^{3}$ and factorized $x^{3}+c^{3}$ into $(x+c)\left(x^{2}-c x+c^{2}\right)$ before also using results about the divisors of a sum of two squares. In the case of Mordell's equation, the factor $N$ would not be seen as a divisor of a sum of squares but as a divisor of $y^{2}-k l b^{2}$, which would lead Mordell to (tacitly) apply the preliminary considerations.

Mordell asserted that the number $N=x^{2}+k a x+k^{2} a^{2}$ is positive ${ }^{26}$ and that " $a$ will be hereafter so chosen that $N$ is odd" [Mordell 1914a, p. 61]. Yet, stated as such, this assumption is problematic because $N$ depends on $a, k$, and $x$, and because it is not clear whether it is actually possible to choose such an adequate $a$. Although Mordell did not comment on these issues, it is possible to infer from the rest of his paper that he meant here that $k$ being fixed, the parameter $a$ is supposed to be so chosen that $N$ is odd for every possible solution $x$ of (1). As for the possibility of making this choice, a large subsequent part of the paper would be devoted to the proof that, when $k \equiv 3 \bmod 4$ (which would be assumed by Mordell at some point), it is possible to find $a$ such that $N \equiv 3 \bmod 4$ for every $x$ : this is evidently sufficient to get the demanded parity of $N$. That said, Mordell did not explain why he made this assumption, and the fact that $N$ is odd never explicitly reappears later in the paper. Apparently, this hypothesis only served to legitimate the use of the Jacobi symbol $(\cdot / N)$, which is defined only for an odd positive integer $N$.

${ }^{25}$ In other words, this result says that if $x$ and $y$ are coprime, and if $q$ is an odd prime divisor of $x^{2}-k y^{2}$, then $(k / q)=1$. Investigations on such divisors had already been made by Leonhard Euler in the middle of the eighteenth century. These investigations and their link with the reciprocity law are described in [Edwards 1983]. See also [Boucard 2013] for related works of Cauchy on quadratic forms $x^{2}+n y^{2}=4 p^{\mu}$. Moreover, note that [Mathews 1892, p. 52] also dealt with divisors of $x^{2}-k y^{2}$, yet in a different way than Mordell.

${ }^{26}$ This can be seen by writing $N=\left(x+\frac{k a}{2}\right)^{2}+\frac{3}{4} k^{2} a^{2}$. 
The next move, indeed, was to affirm that $(k l / N)=1$ if $N$ is prime to $k l b^{2}$, a condition that Mordell then examined by considering separately the coprimality of $N$ with $k, l$, and $b .{ }^{27}$ Thus he first proved, thanks to the hypotheses that $k$ is square-free and prime to $b l$, that $N$ is prime to $k$, and he added that "[a]s to $N$ being prime to $l(\neq \pm 1)$, this is best postponed to the stage when particular values of $l$ are considered; but this will always be the case." [Mordell 1914a, p. 61]. However, Mordell did not return to this question, even when he considered the specific cases $l=2,3$, and 6 later in the article. Let us also note that when he wrote " $l(\neq \pm 1)$," Mordell did not mean to exclude the values \pm 1 for $l$ : rather, it signified that the hypothesis of the coprimality of $N$ and $l$ had to be made only in the non-trivial situations where $l \neq \pm 1$.

He went on, writing that if $N$ is prime to $b$, then

$$
\begin{aligned}
(l / N) & =(k / N)(N / k) \\
& =(-1)^{\frac{1}{4}(k-1)(N-1)} .
\end{aligned}
$$

Mordell did not give the proof of these equalities in detail, only indicating that they were linked with the fact that $(N / k)=1,{ }^{28}$ and that one should treat the case of a positive $k$ after dealing with a negative $k$. Indeed, when $k>0$, the second equality is just the quadratic reciprocity law; furthermore, because $(N / k)=1$, the first one is equivalent to $(l / N)=(k / N)$. But supposing that $N$ is prime to $b$ (and to $l$ ), one has $(k l / N)=1$ according to the above. Therefore $(k / N)(l / N)=1$ and then $(k / N)=(l / N)$ since these two numbers are 1 or -1 . As for the case $k<0$, the result holds similarly, with computations involving $(N /-k),(-k / N)$, and the known value for $(-1 / N)$.

After having written equation (2), Mordell concluded that "[i]f, therefore, values of $a$ and $b$ can be chosen such that for given $k$ and $l,(2)$ is untrue, it will follow that (1) is insoluble" [Mordell 1914a, p. 61]; in particular, he noted that when $l=1$, the initial equation (1) is insoluble when $a$ and $b$ are such that $k$ and $N$ are congruent to 3 modulo 4 . It must be remarked, though, that Mordell's conclusion (for any $l$ ) is questionable: since the identity (2) has been proved under the assumption that $N$ and $b$ are coprime, its negation should imply either that (1) is untrue, or $N$ is not prime to $b$. An explanation of this problem could be that Mordell tacitly supposed that $b$ was chosen to be prime to $N$; however, as he did not evoke or do anything about such a possibility in the rest of the paper, this seems quite unlikely. We will come back to the interpretation of this problem after having analyzed Mordell's next paragraph, which will shed additional light on it.

This paragraph began with the hypothesis that (2) is untrue, an hypothesis that Mordell explained is equivalent to $(k l / N)=-1$. This implied the existence of a prime factor $q$ of $N$ such that $(k l / q)=-1$, and which would also divide $b$ according to the preliminary considerations. Because of equation (1), such a $q$ divides $y$, and Mordell wrote $y=q y_{1}$ and $b=q b_{1}$, which gives

$$
y_{1}^{2}-k l b_{1}^{2}=(x-k a) N / q^{2} .
$$

\footnotetext{
${ }^{27}$ In the course of the preliminary considerations Mordell had proved that if $p$ is an odd prime factor of $x^{2}-k y^{2}$ and if $p$ is prime to $k y$, then $(k / p)=1$. Here, every prime factor $p$ of $N$ divides $y^{2}-k l b^{2}$, so that $(k l / p)=1$ if $N$ is prime to $k l b$ (or, equivalently, to $\left.k l b^{2}\right)$ : this yields $(k l / N)=1$.

${ }^{28}$ Because $N=x^{2}+k a x+k^{2} a^{2}$ is congruent to $x^{2}$ modulo $k$, one has $(N / k)=\left(x^{2} / k\right)=(x / k)^{2}$. This is equal to 1 since $x$ is prime to $k$.
} 
Next, Mordell showed that $q^{2}$ divides $N$, provided two supplementary conditions are assumed, namely that $a$ and $b$ have no common prime factor $Q$ such that $(k l / Q)=-1$, and that $b \not \equiv 0 \bmod 3$ in the case where $(k l / 3)=-1$. It is interesting to see how Mordell introduced these conditions: the idea is that a sufficient condition for $q^{2}$ to divide $N$ is that $q$ and $3 k a x$ are coprime. ${ }^{29}$ Then, as he had done in the case of the coprimality of $N$ and $k l b^{2}$, Mordell examined separately the coprimality of $q$ with $3, k, a$, and $x$. Thus he proved that $q$ is prime to $k$ thanks to the hypotheses made at the beginning of the proof, but he wrote that " $a$ is prime to $q$ if $a$ and $b$ have no common factors of the type $q$," $i$.e. if $a$ and $b$ have no common prime factors $Q$ such that $(k l / Q)=-1$ : this is the first supplementary condition. Let us emphasize that this condition tautologically implies that $q$ and $a$ are coprime, since $q$ is, by its very definition, a factor of $b$ "of the type $q$." Similarly, the second condition was introduced to assure that $q$ is prime to 3: since the prime number $q$ divides $b$ and satisfies $(k l / q)=-1$, it is necessarily prime to 3 under the condition that either $(k l / 3)=+1$ or, in the opposite case, 3 does not divide $b$. Hence the two supplementary conditions were added in the very course of the proof, in order to (tautologically) address two specific technical points. This manner of inserting new hypotheses - that the reader understands to be new hypotheses, and not sufficient conditions which Mordell would prove to be true with the help of the other premises - thus contributes to a certain manner of writing and presenting a demonstration which is quite unsettling at first sight.

After having proved that $q^{2}$ divides $N$, Mordell wrote $y_{1}^{2}-k l b_{1}^{2}=(x-k a) M_{1}$ with $N=M_{1} q^{2}$ and $\left(k l / M_{1}\right)=-1$. He then explained that "continuing this process, we can remove all the factors of $b$ typified by $q$, and finally arrive at an equation of the form

$$
Y^{2}-k l B^{2}=(x-k a) M,
$$

where $(k l / M)=-1$; but $B$ and $M$ have no common factors $q$ such that $(k l / q)=-1$." [Mordell 1914a, p. 62]. This, Mordell wrote, led to a contradiction, which proved that if the equation (2) is untrue and if the two supplementary conditions on $a$ and $b$ are satisfied, then the initial equation (1) is impossible. ${ }^{30}$

This result marked the end of a certain part of Mordell's paper, as it was the last time that Mordell considered unspecified values of the parameter $l$. At this point, all the results that had been proved were valid only under restrictive hypotheses that were not made completely explicit in terms of the parameters $k, l, a, b$. Indeed, what Mordell did so far can be summed up as follows:

1. One considers the equation $y^{2}-k l b^{2}=x^{3}-k^{3} a^{3}(1)$, with the conditions that $k$ is square-free and prime to $b l$, and that $a$ is such that $N=x^{2}+k a x+k^{2} a^{2}$ is odd for every possible solution $x$ of (1). One also supposes that $N$ is prime to $l$.

${ }^{29}$ Since $q^{2}\left(y_{1}^{2}-k l b_{1}^{2}\right)=(x-k a) N$, the number $q^{2}$ divides $N$ if $q$ is prime to $x-k a$. Now, write $N=(x-k a)^{2}+3 k a x$ : as $q$ divides $N$, the coprimality of $q$ with $3 k a x$ is equivalent to that of $q$ with $x-k a$.

30 Just like above, since $(k l / M)=-1$, there exists a prime divisor $q$ of $M$ satisfying $(k l / q)=-1$. Then, as $M$ divides $Y^{2}-k l B^{2}$, the preliminary considerations show that $q$ also divides $B$, whereas $B$ is supposed not to have such a factor. Remark that Mordell's reasoning can be interpreted as a form of infinite descent, even if Mordell neither made any comment on it, nor even introduced this appellation. As recalled in our introduction, the infinite descent played a crucial role in the proof of the finite basis theorem published in [Mordell 1922b]. See [Goldstein 1993, pp. 40-42]. 
2. If $N$ is prime to $b$, then the equation $(l / N)=(-1)^{(k-1)(N-1) / 4}$ (2) is true. Thus if $k$, $l$, and $N$ are such that (2) is untrue, then the equation (1) is insoluble. In particular, when $l=1$, this is the case when $k, N \equiv 3 \bmod 4$.

3. If (2) is untrue, and if one assumes the two supplementary conditions on $a$ and $b$, then the equation (1) is insoluble.

Even if this summary makes all the little technical issues that were left unproved by Mordell disappear, the reader may agree with us that it is still difficult to grasp the general economy of the proof. In particular, what we have numbered 2 and 3 do not form a disjunction of cases: each of them separately yield results about the initial equation (1) that are subjected to specific hypotheses (that $N$ is prime to $b$, and that (2) is untrue, respectively).

Yet, when Mordell passed to the case $l=1$ and formulated the corresponding result, these hypotheses disappeared. Indeed, considering the equation

$$
y^{2}-k b^{2}=x^{3}-k^{3} a^{3},
$$

he supposed that $k$ is square-free, prime to $b$ and congruent to 3 modulo 4 , that $a$ and $b$ have no common prime factors $q$ such that $(k / q)=-1$, and that $b \neq \equiv \bmod 3$ if $(k / 3)=-1$, and he concluded that the equation (3) is insoluble if $a$ and $b$ are such that $N \equiv 3 \bmod 4$.

This conclusion holds because, with the conditions $k, N \equiv 3 \bmod 4$ (and $l=1$ ), the equation (2), which becomes $1=(-1)^{(k-1)(N-1) / 4}$, is untrue: according to the point that we numbered 3, the equation $y^{2}-k b^{2}=x^{3}-k^{3} a^{3}$ is insoluble. Hence one wonders what is the role played by point 2 , for it was not used even in the next cases $l=2,3,6$. In our opinion, its existence suggests that Mordell did not try to make his exposition efficient or minimalist. On the contrary, he seemed to have written his paper following his thoughts, exploring paths even if they led only to partial results, adding new hypotheses along the way, etc.-let us note that such a presentation may be seen as a means to access Mordell's heuristic, even if he did not comment on it. But such a way of writing also becomes a difficulty for the historian faced with reading the paper, both to understand it and to account for it. Yet other features make the reading arduous, such as the absence of astute or fastidious computations.

\subsection{Missing computations and explanations}

Indeed, after having summed up the intermediary result for $l=1$, Mordell turned to the search for values of $a$ and $b$ that force the number $N=x^{2}+k a x+k^{2} a^{2}$ to be congruent to 3 modulo 4, which would lead to an insoluble equation. Since Mordell supposed now that $k \equiv 3 \bmod 4$, it was thus about finding $a$ and $b$ such that

$$
x^{2}-a x+a^{2} \equiv 3 \bmod 4
$$

for every solution $x$ of the equation $y^{2}-k b^{2}=x^{3}-k^{3} a^{3}$ (3). To do so, he considered the different values of $a$ modulo 4 separately. For instance, for $a \equiv-1 \bmod 4$, he affirmed that the values such that $N \equiv 3 \bmod 4$ are $x \equiv 1$ and $x \equiv 2$. Then, reducing the equation (3) modulo 4 gave $y^{2} \equiv-x^{3}-b^{2}$ and Mordell asserted that if $b$ is even then this implies $x \equiv 1 \bmod 4$, 
which is one of the desired values. He concluded: "Hence we have the first insoluble equation $y^{2}=x^{3}-k^{3}(4 a-1)^{3}+4 k b^{2} . "{ }^{31}$ [Mordell 1914a, p. 63].

It is easy to check every point that Mordell left unproved here. For instance, when $b$ is even, one has $b^{2} \equiv 0 \bmod 4$ and so $y^{2} \equiv x^{3}-1$. To see, then, that this implies $x \equiv 1$, one can list all the possible values of squares $y^{2}$ and cubes $x^{3}$ modulo 4 and observe that the only solution to $y^{2} \equiv x^{3}-1$ is given by $x \equiv 1$. Nevertheless, we want to emphasize that Mordell did not explain how he found that $b$ must be even in order to force $x$ to be congruent to 1 . In fact, he proceeded in the same way (and with similar blanks) in the other cases, where the computations were more complicated.

Consider for instance the passage that we copied in Figure 2: it concerns the case $a \equiv 2 \bmod 4$, for which Mordell had previously proved that the values $x$ making $N \equiv 3$ are $x \equiv 1$ and $x \equiv-1$.

$$
\begin{aligned}
& \text { Take } a \equiv 2 \bmod 4 \text { and } b \equiv 1 \bmod 2 \text {, then } x \equiv 1 \bmod 4 \text {, and hence the } \\
& \text { insoluble equation } \\
& \qquad y^{2}=x^{3}-k^{8}(4 a+2)^{3}+k(2 b+1)^{2} .
\end{aligned}
$$

Take $a \equiv 2 \bmod 4$ and $b \equiv 0 \bmod 2$, then $x \equiv 1 \bmod 4$ or even; so if vee can find values of $a$ and $b$ satisfying these congruences, and also such that $x$ cannot be even, ${ }^{*}$ then (3) is insoluble.

Now when $A \equiv 4 \bmod 8$, and

$$
y^{2}=x^{3}+A
$$

admits of even values for $x$, we have $y \equiv 2 \bmod 4$, whence

$$
A \equiv 12 \bmod 16 \text { or } \equiv 4 \bmod 32 \text {, }
$$

i.e.

$$
A=-12 \bmod 32 \text {. }
$$

Hence the values of $a$ and $b$ needed are given by

$$
-k^{3} a^{3}+k b^{2} \equiv-12 \bmod 32,
$$

from which we obtain

$$
a \equiv-k-3 \bmod 8 \text { and } b \equiv 2 \bmod 4,
$$

and hence the insoluble equation

$$
y^{2}=x^{3}-k^{3}(5+8 c-k)^{3}+4 k(2 b+1)^{2} .
$$

Figure 2 - Extract of Mordell's paper (p. 63).

While the first sub-case, with $b \equiv 1 \bmod 2$, looks just like the one we just depicted, the second sub-case, with $b \equiv 0 \bmod 2$, is more puzzling, as the blanks here multiply: why take $A \equiv 4 \bmod 8$ ? Why is it true that $y \equiv 2 \bmod 4$ ? What are the justifications

\footnotetext{
${ }^{31}$ In this equation, the letters $a$ and $b$ obviously do not have the same meaning as above: the previous $a$ is replaced by $4 a-1$ and the previous $b$ is replaced by $2 b$. Such shifts in notations are very frequent in Mordell's text. Moreover, although this was not recalled, the numbers $4 a-1$ and $2 b$ must satisfy the conditions given above, namely that $4 a-1$ and $2 b$ have no common prime factor $q$ such that $(k / q)=-1$ and that $2 b \not \equiv 0 \bmod 3$ if $(k / 3)=-1$ : since Mordell did not rewrite these conditions, one could have thought that the specific, new forms $4 a-1$ and $2 b$ made them tautologically true, but this is not the case.
} 
of the "whence" and the "i.e."? Is it supposed to be straightforward that the solutions of $-k^{3} a^{3}+k b^{2} \equiv-12 \bmod 32$ are $a \equiv-k-3 \bmod 8$ and $b \equiv 2 \bmod 4$ ? Again, it is possible for the reader to check that Mordell's assertions are true-it requires elementary, yet fine and sometimes tedious algebraic manipulations-, but it must be stressed that Mordell left no clue on how he found them in the first place. In the case of the congruence $-k^{3} a^{3}+k b^{2} \equiv-12 \bmod 32$ for example, how did he found the right values for $a$ and $b$ ? Did he make many computations by trial and error, for instance on the values of $b$ and then $a$ modulo a certain number until he found the result? Did he recognize a special congruence that he encountered in some other research publication or a textbook where the solutions were given? Was this congruence well-known to him? Did he know classical methods that allow one to deal with congruences modulo powers of 2 ?

We do not want to address these questions here. Our point is that all the blanks left by Mordell, and which appeared to us when reading his paper in minute detail, bring to light difficulties of reconstituting, from the published article, certain facets of his mathematical practice. Furthermore, the accumulation of such blanks throughout Mordell's paper, as well as the logical weaknesses that we observed above, induce another effect, that of the blurring of the line between what is merely asserted and "what is actually proved," to borrow Cassels' words.

\subsection{Numerical values, generalizations, and a tentative method}

Before passing on the other parts of Mordell's paper, we sum up the end of the first one without describing it in the same amount of detail as above. As he closed the paragraphs dealing with the case $l=1$, Mordell listed numerical values of parameters corresponding to insoluble equations, when $k=-1$ : "we find some of the known insoluble equations $y^{2}=x^{3}+A^{3}-B^{2}$, say, and our conditions become $B \not \equiv 0 \bmod 3$, and $A$ and $B$ have no common prime factor congruent to $3 \bmod 4$. We thus find the insoluble equations $y^{2}+k=x^{3}$ where $k=9,3,12,43,91,99$, and $-k=95,47,39,11,67,53,13,20$." [Mordell 1914a, p. 64]. Mordell remained silent on which were the "known" insoluble equations; in fact, among the numbers he listed, only $3,9,43,91,-47$, and -11 had been treated in some of the works cited in the introduction, more precisely in papers of Pépin [1875, 1882] and De Jonquières $[1878 \mathrm{a}, \mathrm{b}] .^{32}$

After having considered the case $l=1$, Mordell quickly expounded the results he obtained for $l=2,3$, and 6 , indicating that one could follow "the same procedure as before," and he listed his results, which were in the same vein as the ones for $l=1$. For instance, for $l=2$, he stated that the equation $y^{2}=x^{3}-k^{3} a^{3}+2 k b^{2}$ is insoluble if $a \equiv 2+4(-1)^{(k-1) / 2} \bmod 16$ and $b \equiv 2 \bmod 4$; if $k$ is odd, square-free, and prime to $b$; if $b \neq 0 \bmod 3$ in the case where $(k / 3)=1$; and finally if $a$ and $b$ have no common prime factor $q$ such that $(2 k / q)=-1$. In the specific cases where $k= \pm 1$, Mordell listed eight insoluble equations with numerical coefficients when $l=2$, such as $y^{2}-66=x^{3}$ or $y^{2}+98=x^{3}$; he gave two such equations

\footnotetext{
${ }^{32}$ With the help of a computer we have generated a number of the values given by Mordell's formulas, and we found other results than those listed by him. For instance, the case of $y^{2}=x^{3}+(4 a-1)^{3}-4 b^{2}$ yields (among others) the insoluble equations $y^{2}=x^{3}-5$ and $y^{2}=x^{3}+23$. All the supplementary values comprised between -100 and 100 that we computed appear in the section dealing with ideal numbers, expect for $y^{2}=x^{3}-33$. Moreover, remark that the last quotation of Mordell is about the equation $y^{2}+k=x^{3}$ and not $y^{2}-k=x^{3}$. Such (exasperating) substitutions between $k$ and $-k$ repeatedly occur in the paper, for no apparent reason.
} 
for $l=3$, and none for $l=6.33$

The two next paragraphs concerned two other kinds of Diophantine equations linked to $y^{2}-k=x^{3}$. On the one hand, Mordell stated generalizations of the previous results to equations of higher degree such as $y^{2}=x^{2 n+1}-k^{2 n+1} a^{2 n+1}+k b^{2}$, with specific hypotheses on $a, b$, and $k$. On the other hand, he presented a particular method that allowed to prove, in some specific cases, that equations of the form $y^{2}=x^{3}+8 k+5$ are insoluble.

The last section of the first part of Mordell's article concerned "a tentative method for finding solutions of $y^{2}=x^{3}+k$. ." [Mordell 1914a, p. 67]. The idea, which Mordell expounded quite vaguely, consisted in examining, for a given equation, the different possible values of $x$ modulo 8, then modulo 3, 5, 7, etc. For each of the possibilities for $x$ so obtained, the method consisted then in choosing a new modulus $M$ and checking whether $x^{3}+k$ is a quadratic residue modulo $M$. Rejecting the $x$ 's for which this was not the case, this would give a list of possible values of $x$ to be eventually tested "by actual substitution." This method echoes a short note of Cunningham [1905] that Mordell had cited in the introduction. Indeed, Cunningham considered the equation $y^{2}-17=x^{3}$, and explained that looking at the possible unit digits for $y^{2}$ allowed one to restrict the possible values of $x$ modulo 10; after eliminating values of $x$ modulo 100 and 1000 though the inspection of the two (resp. three) last digits of $y^{2}$, Cunningham was able to list the five only solutions $(x, y)$ to the equation with $x<500$ (and $y>0$ ).

Mordell did not give any example there: he just indicated that for $k=-31$, he found that there were no solutions with $x<10^{9}$. We have no clue to how Mordell articulated his tentative method with the other facets of his approach of the equation $y^{2}-k=x^{3}$. For instance, did he use this method to guess what were the possible forms of the parameters corresponding to impossible equations? Did he, on the contrary, use it to verify that the equations he proved to be insoluble had, indeed, no solutions with $x<10^{9}$ ? In any case, at the very end of the paper, as he summed up his results and formulated some conjectures, Mordell listed $y^{2}+31=x^{3}$ among the equations about which "whether or not the equations are insoluble [he] cannot say." [Mordell 1914a, p. 80]. But he also (mistakenly) listed a number of equations having, he thought, an infinite number of integral solutions. Again, Mordell did not explain why he thought so, and it is possible that they corresponded to equations for which the tentative method did not allow him to rule out a great number of possibilities for $x$. To take but one example, he listed $y^{2}+20=x^{3}$ as one of the equations having an infinite number of solutions, whereas it can be proved that it has only one, namely $(6, \pm 14)$.

\subsection{The tip and the hidden part of the computations}

As is reflected in the many questions that we raised throughout this section, the examination of the first part of Mordell's paper highlights a number of missing computations and explanations, whose absence limits our understanding of his activity. Furthermore, these missing elements, together with the dispersion of technical hypotheses in this article, the absence of clear statements of the results, and the apparent flaws of the logical structure of the proof, make it

\footnotetext{
${ }^{33}$ Here again, we computed supplementary results, which are all redundant with the values given somewhere else in Mordell's paper. In particular, Mordell's formulas for $l=6$ do give some numerical results between -100 and 100 , but they all appear either in the case $l=2$ or in the next part, dealing with ideal numbers.
} 
all the more difficult to grasp Mordell's technicalities.

But these issues are not problematic in the same way. Hiding fastidious computations or giving (correct) formulas without explaining how they came into the author's mind, was certainly usual in a research paper published in the beginning of the twentieth century, and it did not hinder our overall understanding of Mordell's proof. Such missing technical details thus appear to be less serious than the vagueness of the logical coherence which we accounted for above, and which may be more likely to jeopardize the general economy of the proof.

Nevertheless, we remark that none of the problems we have listed until now are connected to the definition of the mathematical objects implied in the proofs: the congruences, the Jacobi symbol, or the quadratic reciprocity law. On the contrary, the objects that are central in the second part of the paper suffer from an absence of definition which makes the understanding of this part quite puzzling for a different reason.

\section{Introducing ideal, yet ambiguous, numbers}

Mordell's second approach to the equation $y^{2}-k=x^{3}$ consisted in using what he called "ideal numbers." Unlike the previous approach, the method that he developed here let him find both insoluble equations and equations having a finite number of solutions, for which explicit formulas were provided. While Mordell treated several cases corresponding to different hypotheses on the parameter $k$, the proofs always followed the same pattern. The first step was the factorization of the left-hand side $y^{2}-k$ into $(y+\sqrt{k})(y-\sqrt{k})$, which thus introduced irrational or complex numbers, according to the value of $k$. Handled with the support of "ideal numbers" (whose properties depend on $k$ ), this factorization then helped Mordell write $x$ and $y$ as expressions depending on some new integers $a$ and $b$, which themselves satisfied an identity involving $k$. The examination of this identity eventually led either to specific forms of $k$ corresponding to soluble equations or to impossible equations.

Using numbers of the form $a+b \sqrt{k}$ to solve Diophantine equations was of course not a new idea in 1914. Mordell himself [1914a, p. 67] recalled that Euler had erroneously solved the equation $y^{2}+2=x^{3}$ by considering numbers of the form $a+b \sqrt{-2}$, [Euler 1822, p. 401]. ${ }^{34}$ Mordell also referred to a memoir of Johann Peter Gustav Lejeune-Dirichlet [1828] (cited via the Werke of the latter) and the 1875 paper of Pépin which he already cited in the introduction; their existence, he wrote, made it "very curious that the application of ideal numbers [to the equation $y^{2}-k=x^{3}$ ] has been overlooked." These papers of Dirichlet and Pépin, indeed, contained proofs of (in)solubility of Diophantine equations which involved numbers of the form $a+b \sqrt{k}$, and that of Pépin included an examination of the equation $y^{2}+c z^{2}=x^{m}$, which led to results on some equations $y^{2}-k=x^{3}$, but none of them made use of anything like "ideal numbers."

In fact, these texts of Euler, Dirichlet, and Pépin, together with two other ones which do not involve "ideal numbers," 35 were the only texts cited by Mordell in this part of the

\footnotetext{
${ }^{34}$ The reference given by Mordell is the chapter of Euler's Elements of Algebra devoted to the more general problem of finding $y, z$ such that $a y^{2}+c z^{2}$ is a perfect power. The mistake of Euler was to think that if $(\sqrt{a} y+\sqrt{-c} z)(\sqrt{a} y-\sqrt{-c} z)$ is a $m$-th power (of an integer), then the two factors are necessarily themselves $m$-th powers of complex numbers of the form $\sqrt{a} p+\sqrt{-c} q$. This mistake is now part and parcel of the history of ideal theory. See [Edwards 1980, p. 323] for instance.

${ }^{35}$ They are both tables displaying solutions of the equations $t^{2}-k u^{2}= \pm 1$, [Legendre 1830, Table X], and
} 
paper. In particular, Mordell did not provide any bibliographic reference to these "ideal numbers," and, as he did not define them himself, one difficulty is to understand what was the underlying mathematical notion he had in mind. Indeed, at first sight the appellation "ideal numbers" seems to relate directly to the theory of ideal numbers that had been elaborated by Ernst Eduard Kummer in the 1840s - this theory had been re-expounded in English in Smith's Report on the Theory of Numbers [1860], which Mordell recurrently cited in other papers of the 1910s. Yet Kummer's theory pertained exclusively to cyclotomic integers, which does not correspond to the ideal numbers in Mordell's research. ${ }^{36}$ On the other hand, the "ideal numbers" of Mordell were not "ideals": they were not seen and treated as sets of complex numbers, which disposes of the hypothesis that Mordell conceived his "ideal numbers" by strictly following Richard Dedekind's 1871 definition of ideals. As is well known, the set-theoretical definition of Dedekind was the one that David Hilbert adopted in his 1897 Zahlbericht, and, since this book became the standard reference in the beginning of the twentieth century, one could have expected that Mordell would deal with these "ideals." 37

To go further into this discussion about Mordell's "ideal numbers," we propose now to describe the technique involved in his treatment of the equation $y^{2}-k=x^{3}$. A problem arises at once, however, because several complements are needed to understand this technique fully. However, for the reasons we just explained, we are not in position to adopt Mordell's personal view on "ideal numbers." Therefore we choose to adopt a modern point of view, and to recall definitions and properties about ideals that may be useful when following Mordell. ${ }^{38}$

Being given a square-free integer $k$ and the quadratic field $\mathbf{Q}(\sqrt{k})$, one considers the ring of the algebraic integers of the field, which are the elements that are the roots of monic equations of degree 2 with integral coefficients: this ring is $\mathbf{Z}[\sqrt{k}]$ if $k \equiv 2,3 \bmod 4$, and $\mathbf{Z}\left[\frac{-1+\sqrt{k}}{2}\right]$ if $k \equiv 1 \bmod 4$. The set of the invertible elements (or units) of the ring also depend on $k$. If $k<0$, it is $\{ \pm 1\}$, unless $k=-1$ or $k=-3$, in which cases it is respectively $\{ \pm 1, \pm i\}$ and $\left\{ \pm 1, \pm \rho, \pm \rho^{2}\right\}$, with $\rho=e^{2 i \pi / 3}$. If $k>0$, the units are of the form $\pm \epsilon^{n}$, where $\epsilon$ is an algebraic integer called the fundamental unit, and which can be expressed with the help of a minimal solution of either $t^{2}-k u^{2}= \pm 1$ or $t^{2}-k u^{2}= \pm 4$, according to whether $k \equiv 2,3$ or $k \equiv 1 \bmod 4$. Moreover, an important property is that every non-zero ideal of a ring of algebraic integers can be decomposed in an essentially unique way as a product of prime ideals. ${ }^{39}$ Finally, being given a ring of algebraic integers, the quotient of the set of

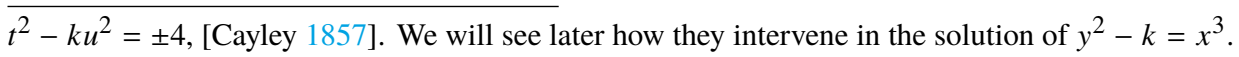

${ }^{36}$ In current notations and terminology, the theory of Kummer deals with algebraic integers of cyclotomic fields $\mathbf{Q}(\alpha)$, where $\alpha$ is a primitive root of unity, while Mordell considers algebraic integers of quadratic fields $\mathbf{Q}(\sqrt{k})$, where $k$ is a non-zero integer. On the history of Kummer's theory, see [Edwards 1980]. Remark that Edwards [1980, p. 328] explains that Kummer wanted to generalize his theory to complex numbers of the form $x+y \sqrt{k}$, but that he never accomplished this generalization.

${ }^{37}$ Moreover, what Mordell does with ideal numbers has nothing in common with Leopold Kronecker's divisor theory. Kummer, Dedekind, Kronecker, and Hilbert are the principal names that are usually associated to the history of ideal theory. See [Edwards 1980; Boniface 2004], as well as [Goldstein and Schappacher 2007b, pp. 75-90]. Finally, see [Schappacher 2005] about the Zahlbericht.

${ }^{38}$ Our decision to use such a modern point of view is also motivated by pedagogical considerations. In particular this choice is not intended to induce any smoothing of the asperities of Mordell's text that we want to share, a smoothing that any modernized presentation could obviously convey. On the issues raised by the writing of (general) history with a current vocabulary, see [Prost 1996, pp. 280-281].

${ }^{39}$ This is the analog of the fundamental theorem of arithmetic in $\mathbf{Z}$. An ideal $I$ is said to be prime if, for any $a, b \in I$, the condition $a b \in I$ implies that either $a \in I$ or $b \in I$. For basic notions of algebraic number theory, see 
the non-zero ideals by that of the non-zero principal ideals can be endowed with a group structure, and it can be proved that this group is finite. It is called the class group and its cardinality is the class number.

\subsection{A first overview of Mordell's ideal numbers}

The first case treated by Mordell in the part dealing with ideal numbers is that of an equation $y^{2}-k=x^{3}$ where $k$ is square-free, negative, congruent to 2 or 3 modulo 4 , and such that the corresponding class number $h$ is not divisible by 3 . Mordell stated that under these hypotheses, $x$ and $2 k$ are coprime 40 and that the equation $y^{2}-k=x^{3}$ has "no solutions unless $k=3 a^{2} \pm 1$, when it has but one." ${ }^{41}$ The proof began with the lines that we reproduce in figure 3.

$$
\begin{aligned}
& \text { Since } y^{2}-k=x^{8} \text {, writing } \theta=\sqrt{ } k \text {, we have } \\
& \qquad(y+\theta)(y-\theta)=x^{9} .
\end{aligned}
$$

But any common factor of $y+\theta$ and $y-\theta$ is a factor of $2 \theta$, and since $x$ is prime to $2 \theta$, it follows that any prime ideal factor of $x$ cannot be a factor both of $y+\theta$ and $y-\theta$; consequently its cube must be a factor either of $y+\theta$ or of $y-\theta$. And since the only units in the domain of $\dot{\theta}$ are \pm 1 , and $\pm i$ when $k=-1$, and as -1 and $\pm i$ can be absorbed in $T_{1}^{8}$, we obtain

$$
\begin{aligned}
& y+\theta=T_{1}^{3}, \\
& y-\theta=T_{2}^{3},
\end{aligned}
$$

where $T_{1}$ and $T_{2}$ are ideal numbers in the domain of $\theta$. But since $h \not \equiv 0 \bmod 8, T_{1}$ and $T_{2}$ are primary numbers, and we can write

$$
T_{1}=a+b \theta, \quad T_{2}=a-b \theta,
$$

and so

$$
y+\theta=(a+b \theta)^{8} \text {, and } x=a^{2}-k b^{2} \text {. }
$$

Figure 3 - Extract of Mordell's paper (p. 68).

As can be seen in this extract, after having written $(y+\theta)(y-\theta)=x^{3}$ with $\theta=\sqrt{k}$, Mordell proved that the cube of any "prime ideal factor" of $x$ is a factor of either $y+\theta$ or $y-\theta ; 42$ although Mordell did not make it clear, it implies that both of these quantities are cubes of ideal numbers up to a unit - this is a consequence of the unique factorization of an ideal into a product of prime ideals, and the analog of the fact that if the product of two coprime integers (in $\mathbf{Z}$ ) is a cube, then the two factors are cubes (up to their sign). But since

\footnotetext{
for instance [Duverney 2010].

${ }^{40}$ This is a technical result that Mordell would use later. That $x$ is odd can be seen by examining the possible values of $y^{2}-k$ and $x^{3}$ modulo 4; the coprimality of $x$ and $k$ comes from the hypothesis that $k$ is square-free.

${ }^{41}$ Because the exponent of $y$ in the equation is even, to any solution $(x, y)$ obviously corresponds another solution $(x,-y)$. Mordell counted these solutions as one, and we will use the same convention.

${ }^{42}$ The proof is written in the two lines following the first centered equation in the extract. The coprimality of $x$ and $2 \theta$ comes from the fact that $x$ is prime to $2 k$ : if $\pi$ divides $x$ and $2 \theta$, then it divides $x$ and $-2 \theta^{2}=2 k$, so that $\pi$ is an unit.
} 
"the only units in the domain of $\theta$ are \pm 1 , and $\pm i$ when $k=-1$," he obtained

$$
y+\theta=T_{1}^{3} \quad \text { and } \quad y-\theta=T_{2}^{3},
$$

where $T_{1}$ and $T_{2}$ are two "ideal numbers," in which the possible units have been "absorbed" since each of the units are cubes.

Mordell deduced from this and from the hypothesis that the class number is not divisible by 3 that $T_{1}$ and $T_{2}$ are "primary numbers," and thus he wrote

$$
T_{1}=a+b \theta \text { and } \quad T_{2}=a-b \theta .
$$

Remark that Mordell's word "primary" seems to be used here to indicate that $T_{1}$ and $T_{2}$ are "actual" complex numbers, as opposed to "ideal" numbers. Indeed, from a current point of view, the equality $y+\theta=T_{1}^{3}$, seen as an equality between ideals, shows that $T_{1}^{3}$ is principal, generated by $y+\theta$. This means that the order of $T_{1}$ in the class group divides 3 ; but since the class number does not divide 3 , the order of $T_{1}$ is necessarily 1 . Hence $T_{1}$ is a principal ideal, generated by a complex number of the form $a+b \theta$, since $k \equiv 2,3 \bmod 4 .{ }^{43}$

According to Dickson's History of the Theory of Numbers [Dickson 1920, p. 296], [Dickson 1923, pp. 203-204], there existed in the nineteenth century a precise notion of "primary complex number," that had been first defined by Gauss for complex integers $a+i b$. This notion, defined by congruences on $a$ and $b$, had been introduced in order to distinguish one specific number between $a+i b$ and its three associate numbers. ${ }^{44}$ It had then been generalized to other kinds of complex numbers; in particular, as can be seen in [Smith 1860, p. 118], Kummer also defined a similar notion of primary complex numbers in the case of cyclotomic integers. Yet, once again, if the terminology may seem to echo this use of "primary number," the examination of the technique shows that Mordell did not use this phrase in this sense, but rather to pass from an ideal number to a complex number.

Once Mordell wrote $T_{1}=a+b \theta$, the conclusion about the equation $y^{2}-k=x^{3}$ was obtained quite quickly: expanding the cube in $y+\theta=(a+b \theta)^{3}$ and identifying the $\theta$-parts gives $b\left(3 a^{2}+k b^{2}\right)=1$, which implies $b=\mp 1$ (since $b$ divides 1 ) and then $-k=3 a^{2} \pm 1$. This result, combined with the fact that $x^{3}=\left(T_{1} T_{2}\right)^{3}$, finally gives $x=a^{2}-k b^{2}=4 a^{2} \pm 1$, which is what Mordell had announced. Mordell then illustrated his result by giving three numerical values of $k$ corresponding to equations with one solution (such as $k=-2$, for which the only solution is $x=3$ and $y=5$ ), as well as thirty values comprised between -100 and 0 that correspond to insoluble equations. For instance, $k=-2$ is equal $-3 a^{2}+1$ for $a=1$, so that the equation $y^{2}+2=x^{3}$ has one solution, given by $x=4 \cdot 1^{2}+1=5$. The insoluble equations are those for which $k$ cannot be written in the form $-3 a^{2} \pm 1$, such as $k=-5$. Let us remark that in these numerical applications Mordell did not raise the problem of selecting only those parameters $k$ for which the class number is not divisible by 3 but, as will be seen in the next section, Mordell knew some tables of Gauss that display (among other data) the class numbers corresponding to many values of $k$, [Gauss 1876, pp. 417-476].

\footnotetext{
${ }^{43}$ We recall that the ideal $T_{1}$ is a priori generated by several algebraic integers. By definition, it is principal if it has only one generator, which can be identified to the ideal, up to a unit.

${ }^{44}$ Numbers are said to be associate numbers if they multiplicatively differ from an unit: in $\mathbf{Z}[i]$, associate numbers are thus of the form $a+i b,-(a+i b), i(a+i b),-i(a+i b)$. Choosing one privileged number among those four was linked to the issue of formulating a biquadratic reciprocity law. For the usual integers, associate numbers are just opposite numbers, and a canonical way to distinguish one of them is to privilege the positive one.
} 
Combining these tables with the formula found by Mordell does yield the listed $k$ 's, except for $k=-90$ which, since it is not square-free, was erroneously given there-this value of $k$ was not listed in the other parts of the paper either.

\subsection{New hypotheses on $k$}

Mordell then announced that similar results hold when $k \equiv 5 \bmod 8$ and $k \neq-3$ (still with $k<0$ ), and he added that it is also the case when $k \equiv 1 \bmod 8$, although "there is great difficulty in dealing with the case of $x$ being even, though our method applies to the odd values of $x$ " [Mordell 1914a, p. 69]. This allusive sentence seems to be related to the fact that when $k \equiv 5 \bmod 8$, the equality $y^{2}-k=x^{3}$ necessarily implies that $x$ is odd, whereas it is not so when $k \equiv 1 \bmod 8$. However Mordell's wording in the passage containing this sentence is so obscure to us that we do not know if he implicitly assumed $x$ to be odd when $k \equiv 1 \bmod 8$, or if he knew an alternative proof as that given below, or if he actually ruled out this possibility for $k$ in this specific technical passage.

In any case, Mordell went on by merely indicating that $y+\theta=(a+b \phi)^{3}$, with $\phi=\frac{-1+\theta}{2}$. As recalled in the beginning of this section, the hypothesis that $k \equiv 1 \bmod 4$ implies that the algebraic integers to consider are of the form $a+b \phi$, and the same proof as above can be achieved to show the existence of integers $a, b$ such that $y+\theta=(a+b \phi)^{3}$, in particular because all the units are cubes (since $k$ is negative and different from -3 ). Mordell also wrote that

$$
x=a^{2}-a b+\frac{1}{4}(1-k) b^{2},
$$

an expression which can be deduced from the equality $x^{3}=(y+\theta)(y-\theta)$. Further, developing the cube $y+\theta=(a+b \phi)^{3}$ and identifying the coefficients of $\theta$ yields

$$
8=b\left[3(2 a-b)^{2}+k b^{2}\right],
$$

another identity given by Mordell. As in the previous case, this identity requires $b$ to be a divisor of 8; Mordell asserted that the only two possibilities are ${ }^{45}$

$$
\begin{aligned}
& b=\mp 1 \quad \text { and } \quad-k=3(2 a \pm 1)^{2} \pm 8, \\
& \text { or } b=\mp 2 \quad \text { and } \quad-k=3(a \pm 1)^{2} \pm 1 \text {. }
\end{aligned}
$$

He finally listed three numerical values of $k$ corresponding to impossible equations, as well as four values corresponding to equations having one or two solutions.

Afterwards, Mordell indicated that all the results he had obtained to this point could be extended to equations of the form $y^{2}-k f^{2}=x^{3}$, where $f$ is such that $x$ is prime to $2 k f$ and " $k$ satisfy the previous conditions" [Mordell 1914a, p. 69] - this new form of the equation thus encompasses the case where the parameter has square factors. For instance, when $k \equiv 1$ or $5 \bmod 8$, Mordell just wrote that

$$
8 f=b\left[3(2 a-b)^{2}+k b^{2}\right] \text { and } x=a^{2}-a b+\frac{1}{4}(1-k) b^{2},
$$

which, indeed, can be obtained by similar considerations as those above. He then gave examples of numerical values of $k f^{2}$ obtained for specified values of $f$. As usual, and as it can be observed in figure 4, Mordell did not provide any of the underlying computations.

\footnotetext{
${ }^{45}$ It is easy to see that the possibilities $b= \pm 4$ and $b= \pm 8$ lead to an impossible equality $8=b\left[3(2 a-b)^{2}+k b^{2}\right]$.
} 
In particular, when $f=4$, and $k \equiv 2,3 \bmod 4, x$ is prime to $2 k$, whence $4=b\left(3 a^{2}+k b^{2}\right)$, which is easily seen to require $b=\mp 1$ and $-k=3 a^{2} \pm 4$. Thus we have no solutions of $y^{2}+16 k=x^{8}$ for $k=1,2,5,6$.

Or, again, when $k \equiv 5 \bmod 8$, and $f=2, x$ is prime to $2 k$, hence

$$
\text { hence, if } \begin{aligned}
b & =\mp 1, & -k & =3(2 a \pm 1)^{2} \pm 16, \\
b & =\mp 2, & -k & =3(a \pm 1)^{2} \pm 2, \\
b & =-4, & -4 k & =3(a+2)^{2}+1 .
\end{aligned}
$$

Thus, for

$$
y^{2}+4 k=x^{8} \text {. }
$$

$$
\begin{array}{ccl}
k=11 & \text { gives only } & x=5, y=9 \\
k=19 & n & x=5, y=7, \text { and } x=101, y=1015
\end{array}
$$

Figure 4 - Extract of Mordell's paper (p. 69-70).

\subsection{Taking the units into account}

Mordell went on: "The simplicity of these results is due to the fact that the only units are \pm 1 , except for the determinants -1 , where no inconvenience is caused, and -3 which was excluded from the discussion. We have, however, interesting results when the units must be taken into account." [Mordell 1914a, p. 70]. Accordingly, at this point he considered the equation $y^{2}-k f^{2}=x^{3}$ with $f$ such that $x$ is prime to $2 k f, k$ free from square factors, positive, and such that the class number is not divisible by 3 .

As explained above, because $k>0$, the units are now of the form $\pm \epsilon^{n}$, where the fundamental unit $\epsilon$ can be expressed with the help of a minimal solution of either $t^{2}-k u^{2}= \pm 1$ or $t^{2}-k u^{2}= \pm 4$ according to whether $k \equiv 2,3 \bmod 4$ or $k \equiv 1 \bmod 4$.

In the first case, Mordell noted $(T, U)$ what he called the solution of $t^{2}-k u^{2}=1$ having the least non-zero value of $u$-Mordell seemingly did not privilege the positive solutions, but rather thought of $U$ up to its sign ${ }^{46}$ - and he asserted (in a very vague passage) that $x=a^{2}-k b^{2}$ and that either

$$
y+f \sqrt{k}=(a+b \sqrt{k})^{3} \quad \text { or } \quad y+f \sqrt{k}=(T+U \sqrt{k})(a+b \sqrt{k})^{3} .
$$

We will not enter into the details to explain this point, and we just mention that these identities can be deduced from the fact that $y+f \sqrt{k}$ is necessarily of the form $\pm \epsilon^{n}(a+b \sqrt{k})^{3}$, an equality obtained just like above. The idea, then, is to link $\epsilon$ with $T+U \sqrt{k}$ on the one hand, and to deal with the absorption of $\pm \epsilon^{n}$ into the cube on the other hand.

The case of $y+f \sqrt{k}=(a+b \sqrt{k})^{3}$ yields exactly the same results as above, which certainly explains why Mordell did not take it into account as he carried on with his proof. As for the possibility $y+f \sqrt{k}=(T+U \sqrt{k})(a+b \sqrt{k})^{3}$, he equated the $\sqrt{k}$-parts of this identity to obtain

$$
f=U\left(a^{3}+3 k a b^{2}\right)+T\left(3 a^{2} b+k b^{3}\right),
$$

${ }^{46}$ This can be inferred from [Mordell 1969, p. 242], where, in the same proof, $U$ is replaced by $\pm U$. 
and found conditions on $k f^{2}$ and $U$ making this identity impossible. For instance, supposing that $k f^{2} \equiv 4 \bmod 9$, he showed through lengthy and ingenious computations that $U$ could not be congruent to 0 mod 9. From this he concluded that the equation $y^{2}-k f^{2}=x^{3}$ is insoluble under the hypotheses written at the beginning of this subsection, as well as $k f^{2} \equiv 4$ and $U \equiv 0 \bmod 9$. In the same way, he also treated the cases $k f^{2} \equiv-4, \pm 7$ and \pm 3 modulo 9 , which, together with certain congruences on $U$ or $T$, yielded impossible equations. ${ }^{47}$

Several numerical values of $k f^{2}$ were then given by Mordell without any comment. We note, however, that their computation must have rested on the knowledge of the minimal solutions of $t^{2}-k u^{2}=1$ : Mordell's reference for this was a table of Legendre's displaying these solutions for every positive, square-free $k$ comprised between 2 and 1003, [Legendre 1830, Table X].

Mordell also treated the case $k \equiv 1 \bmod 4$. The method and computations were very similar to the previous case, the main difference being that the starting point was to write

$$
\begin{aligned}
y+f \sqrt{k} & =\left[T_{1}+\frac{U_{1}}{2}(1+\sqrt{k})\right]\left[a_{1}+\frac{b_{1}}{2}(1+\sqrt{k})\right]^{3}, \\
x & =a_{1}^{2}+a_{1} b_{1}+\frac{1}{4}(1-k) b_{1}^{2},
\end{aligned}
$$

for some integers $a_{1}, b_{1}$, and $T_{1}, U_{1}$ such that $(T, U)=\left(-2 T_{1}-U_{1},-U_{1}\right)$ is a minimal solution of $t^{2}-k u^{2}= \pm 4$. The results and the computations were in the same vein: among other cases, Mordell showed that the equation $y^{2}-k f^{2}=x^{3}$ is impossible under the supplementary hypotheses $k f^{2} \equiv 4$ and $U \equiv 0 \bmod 9$. Numerical instances of $k f^{2}$ were also provided, the reference for knowing the minimal solutions of $t^{2}-k u^{2}= \pm 4$ being now a table of Cayley's, [Cayley 1857].

Mordell closed this part on ideal numbers with a short paragraph in which he illustrated how other results could be found similarly, by considering values of $k f^{2}$ modulo 7 instead of modulo 9. After having expounded a series of equalities and congruences serving as elements of proof, he finally asserted that $y^{2}-k f^{2}=x^{3}$ has no solution when $k \equiv 1,2,3(\bmod 4)$, $k f^{2} \equiv 4(\bmod 7)$ and $U \equiv 0(\bmod 7)$. No numerical value was listed there-in [Mordell 1969], Mordell exemplified these results by providing $k=159$ and $f=1$ : these values were probably not given in the 1914 paper because Mordell focused there on the values of parameters between -100 and 100 .

\subsection{A certain practice of ideal numbers}

As with the image of the first part of Mordell's article, centered on the quadratic reciprocity law, the second one contains many unexplained points, related to the computations and to obscure arguments. A striking difference, though, is that if there was no doubt about the definition of the mathematical objects and notions implied in the first part, this is not the case with the "ideal numbers," which were essential for the proofs of the second part. It thus creates another, more serious, kind of difficulty for anyone who tries to understand Mordell's mathematics, and who might hesitate between two conceptions of "ideal numbers."

\footnotetext{
${ }^{47}$ Once again, we have no clue of how Mordell ended up considering these specific values of the modulus and these possibilities for $k f^{2}$, and we are here reduced to simply check (and complete) his computations.
} 
As we already noticed, the phrase "ideal numbers" seems to refer to the works of Kummer on cyclotomic integers which Mordell could have known through Smith's Report. At the same time, these ideal numbers were not sets of algebraic integers, but numbers, as can be seen in their very designation and in equalities such as $y+\theta=T_{1}^{3}$, where the left-hand side is a complex number and the right-hand side (the cube of) an ideal number. Nevertheless, the properties that were used by Mordell and the general organization of the whole part, based on the knowledge of the different forms of algebraic integers and units according to the value of $k$, point to results that had been proved by Dedekind and that were included in Hilbert's Zahlbericht and its descendants, such as the book of Julius Sommer which was mainly devoted to quadratic fields [Sommer 1907]. Hence Mordell's practice of ideal numbers consisted in a certain interplay between results that were well-known (or sufficiently well-known not to deserve any bibliographic reference) and usages that were quite personal and could even be misleading, such as in the case of "primary complex numbers."

This particular way of dealing with ideal numbers proves that Mordell did not try to fit in any standard frame of the time-such as that provided by the Zahlbericht - which can be connected to his self-education in number theory. But it also emphasizes the caution with which the modern reader has to reconstruct Mordell's research, without misrepresenting its particularities, when compared either to a current point of view or to the practice of some of his contemporaries.

\section{The use of the arithmetical theory of binary cubic forms}

The third and last part of Mordell's paper was based on the arithmetical theory of binary cubic forms, ${ }^{48}$ about which, Mordell explained, "[a]1l that we need is contained in a paper by Arndt," referring to a paper of Friedrich Arndt published more than fifty years before, [Arndt 1857]. This is the only bibliographical reference that Mordell gave about cubic forms, and he only cited it once, in the quotation we just gave. Thus he did not make explicit which results he extracted from it, nor did he specify when he used these results. Actually, we will see that Mordell used pieces of the theory of cubic forms which are not to be found in Arndt's paper, and which may thus be pieces that Mordell considered as elementary or sufficiently well-known for his readers. ${ }^{49}$

Many variations of notations, terminology, and numerical conventions existed among the works of the mathematicians that Dickson's History of the Theory of Numbers listed as the contributors of the theory of binary cubic forms, such as Eisenstein, Arndt, Cayley, Hermite, Pépin, Poincaré, and Mathews, [Dickson 1923, pp. 255-258]. For his part, Mordell denoted a cubic form $(a, b, c, d)=a x^{3}+3 b x^{2} y+3 c x y^{2}+d y^{3}$, and he defined its Hessian to be the quadratic form $\left(b^{2}-a c, \frac{b c-a d}{2}, c^{2}-b d\right)=\left(b^{2}-a c\right) x^{2}+(b c-a d) x y+\left(c^{2}-b d\right) y^{2}$. The determinant $D$ of the cubic, then, is equal to four times the determinant of the Hessian:

\footnotetext{
${ }^{48}$ The adjective "arithmetical," which was used by Mordell, refers to the fact that all the cubic forms at stake have integral coefficients.

${ }^{49}$ According to the History of the Theory of Numbers of Dickson, the theory of binary cubic forms had been launched by Gotthold Eisenstein with a series of papers published in 1844, which Arndt did not know when he first tackled the same theory in the beginning of the 1850s, [Dickson 1923, pp. 255-258]. In the 1857 paper cited by Mordell, however, Arndt did refer to the works of Eisenstein and he listed some of their defects. He also explained that his aim was to simplify some of his own past research on cubic forms, [Arndt 1857, p. 309]. About Eisenstein, see [Schappacher 1998].
} 
$D=(b c-a d)^{2}-4\left(b^{2}-a c\right)\left(c^{2}-b d\right)$. These conventions did not match those of Arndt, who rather considered the quadratic form $2\left(b^{2}-a c\right) x^{2}+2(b c-a d) x y+2\left(c^{2}-b d\right) y^{2}$, which he called the "characteristic" of the cubic. Actually, among the references listed by Dickson on this topic, only Cayley and Mathews used the word "Hessian"; their numerical conventions, however, were different than those of Mordell..$^{50}$

A notion which was central, but not recalled, in Mordell's work is that of the proper representation of a number by a cubic form: an integer $n$ is said to be properly represented by a form $\varphi$ if there exist coprime integers $x, y$ such that $n=\varphi(x, y)$. An elementary property, then, is that two forms (properly) represent the same numbers if they are properly equivalent, i.e. if one of them can be transformed into the other by a linear change of variables with integral coefficients and determinant equal to 1 .

Further, invariants and covariants can be associated to a cubic form. Invariants are polynomials in the coefficients of a form, say $\varphi$, that are unaltered when a linear change of variables with determinant 1 is operated on $\varphi$; covariants are forms whose coefficients are polynomials of the coefficients of $\varphi$, and which are also unaltered by unitary linear changes of variables. ${ }^{51}$ For instance, the determinant $D$ of a cubic form $\varphi$ is an invariant, and the Hessian $H$ is a (quadratic) covariant. Mordell also considered a cubic covariant $T$, which is linked to the previous objects by the relation

$$
T^{2}-D \varphi^{2}=4 H^{3},
$$

called the syzygy of the cubic form. ${ }^{52}$ Although Mordell just used the name "syzygy" without writing down the equality itself or pointing to a bibliographic reference, the syzygy was absolutely central in the approach of the Diophantine equation he was interested in, for it allowed him to link the solutions of the equation with the representations of certain numbers by cubic forms.

\subsection{Linking cubic forms and the solutions of Mordell's equation}

Indeed, Mordell considered the equation $y^{2}-k f^{2}=x^{3}$, and he supposed that $k$ is square-free and that $f$ is such that $x$ is prime to $2 k f$ : as before, this means that $f$ is such that every

\footnotetext{
${ }^{50}$ See [Cayley and Hermite 1857; Mathews 1891] for instance. According to [Crilly 2006, p. 197], the word "Hessian" had been proposed by Sylvester in the beginning of the 1850s. If Mordell's terminology is the same as that of Cayley and Mathews, his numerical conventions are rather those of Hermite. Whatever the chosen convention and terminology were, the consideration of associated quadratic forms was crucial for those who researched cubics, because it created a bridge between the theory of cubic forms (and, in particular, their classification) and that of quadratic forms, which had been extensively studied since Gauss's Disquisitiones Arithmeticae (1801). About quadratic forms in the Disquisitiones, see [Goldstein and Schappacher 2007a, pp. 8-13]. Finally, note that all the elements about quadratic forms that would be used by Mordell appear in Mathews' Theory of Numbers, and that Pépin [1875] made use of the theory of quadratic forms to tackle the equation $x^{2}+n y^{2}=z^{m}$.

${ }^{51}$ More generally, invariants and covariants can be defined for forms with non-necessarily integral coefficients, by the invariance up to a power of the determinant of any linear change of variables (with any non-zero determinant). About the history of invariant theory, see [Fisher 1966; Crilly 1988; Parshall Hunger 1989].

${ }^{52}$ The cubic covariant $T$ is defined by $T=\left(a^{2} d+2 b^{3}-3 a b c\right) x^{3}+3\left(b^{2} c+a b d-2 a c^{2}\right) x^{2} y+3\left(2 b^{2} d-b c^{2}-\right.$ $a c d) x y^{2}+\left(3 b c d-a d^{2}-2 c^{3}\right) y^{3}$. The coefficients of $T$ can be expressed with those of $\varphi$ and of the Hessian $(F, G, H)$. For instance, the coefficient of $x^{3}$ is $b F-a G$. The cubic covariant $T$ and, a fortiori, the syzygy are not to be found in Arndt's 1857 paper. According to [Meyer 1899, p. 351], it had been found and proved by Cayley in his Fifth Memoir upon Quantics, [Cayley 1858].
} 
$x$ satisfying the equation is prime to $2 k f$. The first move was to prove that this equation has solutions if and only if $f$ can be properly represented by a binary cubic of determinant $4 k$, a condition that Mordell asserted to be equivalent to the existence of binary cubics with determinant $4 k$ and whose first coefficient is $f .53$

The syzygy helped Mordell prove that if a cubic form $\varphi$ with determinant $4 k$ and first coefficient $f$ exists, then the equation $y^{2}-k f^{2}=x^{3}$ has at least one solution. He explained that this comes from the identification of the coefficients of $x^{6}$ in the syzygy of $\varphi$, which, indeed, yields

$$
(b F-f G)^{2}-k f^{2}=F^{3},
$$

where $F$ and $G$ are the two first coefficients of the Hessian of $\varphi$. Hence $(F, b F-f G)$ is a solution of $y^{2}-k f^{2}=x^{3}$.

Reciprocally, Mordell showed how to construct a cubic of determinant $4 k$ and first coefficient $f$, under the hypothesis that there exists integers $F$ and $q$ such that $q^{2}-k f^{2}=F^{3}$. For this, he first asserted that there exists a quadratic form $(F, G, H)$ with determinant $k .{ }^{54}$ Then he constructed a cubic form $(f, b, c, d)$ whose Hessian is the quadratic form $(F, G, H)$ - the determinant of the cubic is then equal to what is required, since it is four times that of the Hessian, i.e. $4 k$. Mordell's construction actually consisted only in defining

$$
b=\frac{1}{F}(q+G f), \quad c=\frac{1}{F^{2}}\left(k f+2 q G+f G^{2}\right), \quad d=\frac{1}{F^{3}}\left(k q+3 k f G+3 q G^{2}+f G^{3}\right) .
$$

He just stated that it was easy to see that $b$ is an integer, but he proved (with the help of ingenious but elementary manipulations modulo $F^{2}$ ) that $c$ is an integer as well, and announced that the proof for $d$ was similar. ${ }^{55}$ Although Mordell did not comment on this point, it is interesting to note that the problem of constructing a cubic form having a prescribed Hessian had been tackled in the paper of Arndt cited by Mordell, [Arndt 1857, pp. 312-313]. More precisely, Mordell's definition of $b, c, d$ and his (pieces of) proof that these numbers are integers can be found almost verbatim in Arndt's article.

This ended the demonstration of the equivalence linking the solutions of $y^{2}-k f^{2}=x^{3}$ with the representations of $f$ by binary cubics of determinant $4 k$. The rest of Mordell's paper consisted in using this equivalence to prove that, under certain supplementary hypotheses, there was either none or a finite number of solutions of the equation.

\subsection{A faulty proof?}

Consider for instance the extract displayed in figure 5, which just followed the proof of the previous equivalence.

${ }^{53}$ If $f$ is the first coefficient of a form $\varphi$, then $\varphi(1,0)=f$ is a proper representation of $f$. Conversely, if $f=\varphi(u, v)$ is a proper representation of $f$, it is possible (thanks to Bezout's identity between $u$ and $v$ ) to define a substitution with determinant 1 mapping $(u, v)$ on $(1,0)$. If $\varphi$ is transformed into $\varphi^{\prime}$ by this substitution, then $f=\varphi(u, v)=\varphi^{\prime}(1,0)$, which proves that $f$ is the coefficient of $x^{3}$ in $\varphi^{\prime}$.

${ }^{54}$ Mordell only suggested that $G$ was supposed to be so chosen that $f G \equiv-q \bmod F$ : this is possible because, as every number $x$ satisfying $y^{2}-k f^{2}=x^{3}$ is supposed to be prime to $f$, the numbers $f$ and $F$ are coprime. The form $(F, G, H)$ being supposed to have its determinant equal to $k$, the last coefficient $H$ must be defined by the relation $G^{2}-F H=k$. Thus there remains to show that $F$ divides $G^{2}-k$. But $f^{2}\left(G^{2}-k\right) \equiv q^{2}-k f^{2} \equiv 0 \bmod F$, and since $\operatorname{gcd}(f, F)=1$, this proves that $F$ divides $G^{2}-k$.

${ }^{55}$ The same techniques indeed apply to $d$. That $b$ is an integer comes from the fact that $G$ has been defined 
13. When $k$ is negative, and the class number: (now and hereafter we mean by this the number of properly primitive classes of binary quadratics of determinant $k$ ) is not divisible by 3 , the binary cubics are comprised in the class $(0,1,0, k)$, and hence we have either none or a limited number of proper representations of $f$, and hence none or a limited number of solutions of our original equation.

Figure 5 - Extract of Mordell's paper (p. 75).

This laconic paragraph concerned the case where $k<0$ and where what Mordell called the class number (that we denote by $h$ ) is not divisible by $3 .{ }^{56}$ To fully understand this paragraph, we see that three implications are to be deciphered. We temporarily leave the first two and focus on the third, which states that the number of solutions of the equation $y^{2}-k f^{2}=x^{3}$ is at most finite if the number of proper representations of $f$ (by cubics with determinant $4 k$ ) is at most finite.

At first sight, this may seem to follow directly from the equivalence that we accounted for above. Yet we claim that important clarifications have to be made in order to derive the implication. Indeed, the previous equivalence links the existence of representations of $f$ by cubics of determinant $4 k$ with that of solutions of $y^{2}-k f^{2}=x^{3}$, but Mordell did not explicitly quantify this link, which is crucial to relate the finiteness of both objects: a priori, to each representation of $f$ could correspond infinitely many solutions. This is actually not the case, and it can be proved with Mordell's tools and constructions described before, albeit with a more careful handling. Let us also remark additionally that another point is unclear: what does it mean to count the representations of $f$ ? As will be seen, it seems that Mordell had in mind a counting up to proper equivalence. In other words, two expressions $f=\varphi(x, y)$ and $f=\varphi^{\prime}\left(x^{\prime}, y^{\prime}\right)$ are counted as one representation of $f$ if $\varphi^{\prime}\left(x^{\prime}, y^{\prime}\right)$ is obtained from $\varphi(x, y)$ by a linear substitution of determinant $1 .{ }^{57}$

Let us now reinterpret what we have seen so far. Being given a cubic form $\varphi$ with determinant $4 k$, the syzygy $T^{2}-4 k \varphi^{2}=4 H^{3}$ proves that the pair of integers $(H(\xi, \eta), \pm T(\xi, \eta) / 2)$ is a solution of $y^{2}-k f^{2}=x^{3}$ for every couple of coprime integers $(\xi, \eta)$ such that $\varphi(\xi, \eta)=f .{ }^{58}$

through the congruence $f G \equiv-q \bmod F$. The form $(f, b, c, d)$ being then completely defined, to check that its Hessian is $(F, G, H)$ is a mere computation.

${ }^{56} \mathrm{We}$ recall that a quadratic form $(a, b, c)$ is said to be primitive if $\operatorname{gcd}(a, b, c)=1$, and properly primitive if, additionally, $\operatorname{gcd}(a, 2 b, c)=1$. Moreover, the label "class number" usually refers to the number of equivalence classes of (non necessarily properly primitive) quadratic forms, a number that Gauss had proved to be finite in his Disquisitiones. Since the determinant $k$ is square-free, the number of classes of properly primitive forms is either equal to the number of classes of forms, or to its double, according to whether $k \equiv 2,3 \bmod 4$ or $k \equiv 1 \bmod 4$. Gauss had defined a law of composition for quadratic forms which, in turn, defines a law of composition of classes of quadratic forms: in modern parlance, this makes the set of equivalence classes a group, whose order is the class number.

${ }^{57}$ For instance, $x^{2}+y^{2}$ and $x^{2}+2 x y+2 y^{2}$ are properly equivalent by the transformation $(x, y) \mapsto(x+y, y)$. This implies that $5=1^{2}+2^{2}$ and $5=(-1)^{2}+2 \cdot(-1) \cdot 2+2 \cdot 2^{2}$ are counted as one representation of the number 5. Curiously, the number of representations of an integer by forms of given determinant does not seem to have been defined in the references on forms that we have read.

${ }^{58}$ Remark that the syzygy implies that $T(\xi, \eta)$ is even for any $\xi, \eta$. Moreover, the couples $(\xi, \eta)$ such that $\varphi(\xi, \eta)=-f$ give solutions to the equation too. But it can easily be seen that the solution $(H(\xi, \eta), T(\xi, \eta) / 2)$ associated to such a couple is equal to $(H(-\xi,-\eta),-T(-\xi,-\eta) / 2)$, which is one of the solution associated to 
Reciprocally (and this is what Mordell did not clarify), every solution $(u, v)$ of the equation $y^{2}-k f^{2}=x^{3}$ can be written $(u, v)=(H(\xi, \eta), \pm T(\xi, \eta) / 2)$ for some cubic $\varphi$ of determinant $4 k$ and some coprime integers $\xi, \eta$ such that $\varphi(\xi, \eta)=f$. Indeed, it is possible (just as Mordell did) to construct a quadratic form $H$ of determinant $k$ and first coefficient $u$ (so that $u=H(1,0)$ ), and then a cubic form $\varphi$ of determinant $4 k$, with first coefficient $f$ and having the previous quadratic form as its Hessian. Then the syzygy gives $\left(\frac{T(1,0)}{2}\right)^{2}-k f^{2}=u^{3}$, but as $v^{2}-k f^{2}=u^{3}$, this implies $v= \pm T(1,0) / 2$. Therefore we proved that the set of the solutions of $y^{2}-k f^{2}=x^{3}$ can be written

$$
\mathscr{S}=\left\{\left(H_{\varphi}(\xi, \eta), \pm \frac{T_{\varphi}(\xi, \eta)}{2}\right) \mid \varphi \in C_{4 k},(\xi, \eta) \in \mathbf{Z}^{2}, \operatorname{gcd}(\xi, \eta)=1, \varphi(\xi, \eta)=f\right\}
$$

where $C_{4 k}$ is the set of cubic forms with determinant $4 k$. Now, if two cubics are properly equivalent, so are their Hessians and their cubic covariants (via the same linear substitution), which thus describe the same images. In other words, we can write

$$
\mathscr{S}=\left\{\left(H_{\varphi}(\xi, \eta), \pm \frac{T_{\varphi}(\xi, \eta)}{2}\right) \mid[\varphi] \in C_{4 k} / \sim,(\xi, \eta) \in \mathbf{Z}^{2}, \operatorname{gcd}(\xi, \eta)=1, \varphi(\xi, \eta)=f\right\},
$$

where $\sim$ denotes the proper equivalence of cubics and $[\varphi]$ the equivalence class of $\varphi$. This last expression now allows us to understand Mordell's statement easily: if the number of proper representations of $f$ is finite (or equal to 0 ), then the number of cubics $\varphi$ and integers $\xi, \eta$ parameterizing $\mathscr{S}$ is finite (or empty), so that $\mathscr{S}$ itself is finite (or empty).

Note that a theorem that Axel Thue had proved in a paper of 1909 states that for any binary form $\varphi$ of degree greater that 2 and any non-zero integer $m$, the equation $\varphi(x, y)=m$ has a finite number of solutions, [Thue 1909]. Combined with the fact that $C_{4 k} / \sim$ is finite-this was the final result of the 1857 paper of Arndt (p. 321)-Thue's theorem could have helped Mordell see that $y^{2}-k f^{2}=x^{3}$ always has a finite number of solutions. He observed this himself a few years later, in a short note dated 1918 and published in 1920, [Mordell 1920]. ${ }^{59}$ Instead, Mordell had to find specific hypotheses under which it is possible to prove that for a given cubic form $\varphi$, the equation $\varphi(x, y)=f$ has a finite number of solutions.

The first set of supplementary hypotheses was the one that we already described and that appeared in the paragraph reproduced in figure 5: the parameter $k$ was chosen to be negative and the class number of properly primitive quadratic forms $h$ was supposed not to be divisible by 3 . Why do these hypotheses imply the finiteness of the number of representations of $f$ by cubics of determinant $4 k$ ?

the representation $f=\varphi(-\xi,-\eta)$. The uncertainty of the sign of the second coordinate of the solutions of $y^{2}-k f^{2}=x^{3}$ comes from the fact that the exponent of $y$ in the equation is even. As already remarked, Mordell used to talk about unique solutions, thus aiming at solutions $(x, y)$ with $y>0$. However it is quite uneasy, in our reconstruction, to introduce such solutions, for nothing is known about the sign of $T(\xi, \eta)$ in general. That being said, taking into account both of the sibling solutions $(x, y)$ and $(x,-y)$ does not change anything about the possible finiteness of the set of the solutions.

${ }^{59}$ Mordell's ignorance of Thue's paper has been later integrated to the usual story of the equation $y^{2}-k=x^{3}$, by Mordell himself and by others: see for instance [Mordell 1971, p. 957] or [Cassels 1986, p. 35]. About Thue's research, especially on his famous theorem on Diophantine approximation and its consequences, see [Goldstein 2015]. According to [Dickson 1920, p. 538], Edmund Landau also used Thue's theorem in a article published in 1920 to prove that $y^{2}+2=x^{3}$ has a finite number of solutions. 
First, Mordell asserted that these hypotheses imply that every cubic form with determinant $4 k$ (and properly representing $f$ ) is properly equivalent to $(0,1,0, k)$. This is true because, on the one hand, for such a cubic $\varphi$, its Hessian $H$ is a properly primitive quadratic form, and its order is 1 or $3 .^{60}$ But since the class number $h$ does not divide 3 , the class of $H$ is necessarily of order 1, which means that $H$ is equivalent to the principal form $(1,0,-k)$. On the other hand, the cubic $(0,1,0, k)$ also has $(1,0,-k)$ as its Hessian. Now, according to a result stated by Arndt in his 1857 paper (p. 318), as $k<0$, there is only one class of cubic forms having $(1,0,-k)$ as its Hessian: this proves that $\varphi$ is equivalent to $(0,1,0, k)$.

This easily implies the finiteness of the number of proper representations of $f$. Indeed, since there is only one class of cubic forms to consider, namely that of $(0,1,0, k)$, suppose that $f=(0,1,0, k)(u, v)=3 u^{2} v+k v^{3}$ is a representation of $f$ by this form. Then $v$ divides $f$, which gives a finite number of possibilities for $v$, which, in turn, yields a finite number of possibilities for $u$. As explained above, this eventually proved that if $k<0$ and $h \not \equiv 0 \bmod 3$, the equation $y^{2}-k f^{2}=x^{3}$ has either none or a finite number of solutions.

The amount of detail that we just gave to explain Mordell's sentence reproduced in figure 6 displays at least two kinds of difficulties of reading. First there is obviously the issue of the basic knowledge of any reader faced with Mordell's paper (especially on cubic forms, here), an issue that is well illustrated by our two last paragraphs. Indeed, our intervention there consisted only in completing Mordell's steps, giving intermediate results coming from different sources; if the level of detail that we chose is the one that we thought to be adequate for our own readership and for the format of the present article, that of Mordell may well have been sufficient for his contemporaries readers. The situation is different with the first part of our explanations, about the link between the solutions of $y^{2}-k f^{2}=x^{3}$ and the representations of $f$. For this point, indeed, we intervened because we observed that Mordell's argument, as it was presented, was not complete since it did not seem to take into account the finiteness of the objects. Thus it was not about completing a proof with a series of missing intermediate results, but rather about overcoming a proof that was faulty when reading Mordell stricto sensu: the seriousness and the possible consequences of the latter issue are considerably greater than those of the former. But our main point is that this indecision about whether Mordell's proof was right or not is in great part due to his elliptic writing. In other words we are faced here with a case where the writing of the argument crucially impacts its content, and blurs the line between a solid and a faulty proof.

\subsection{The other cases}

Three other sets of hypotheses assuring the finiteness of the number of representations of $f$ by binary cubics of determinant $4 k$ were then considered by Mordell. The first one consisted in supposing that $k>0$ and that the class number $h$ is not divisible by 3 . However, from laconic, Mordell became sibylline, as his explanations were even more reduced than in the first case (see figure 6).

${ }^{60}$ To prove that the Hessian is properly primitive is a mere computation: the primitiveness comes from the hypothesis that $k$ is square-free, and the properly primitiveness can be proved with the syzygy and the assumption that every $x$ satisfying $y^{2}-k f^{2}=x^{3}$ is prime to $2 k f$. The result stating that Hessians of cubic forms are of order 1 or 3 can be found (for $k<0$ ) in [Cayley and Hermite 1857, p. 85]. Arndt's paper does not contain it. Instead of talking about the order of a (class of) form(s), mathematicians of the time would say that Hessians of cubic forms produce the principal form $(1,0,-k)$ (which is the neutral element in the class group) by triplication. 
When $k$ is positive, there are three classes of binary cubics corresponding to our given Hessian, and when the class number is not divisible by 3 , only one of the classes consists of reducible cubics, while the other two are improperly equivalent, and it suffices to consider only one of them.

Figure 6 - Extract of Mordell's paper (p. 75-76).

We will not fill the gaps as we did before, but we want to stress that, here too, much work has to be done in order to complete Mordell's reasoning.

In the two last cases, Mordell exchanged the hypothesis on the class number with hypotheses on what he called the index of irregularity of $k$, a number that was usually called "exponent of irregularity." Indeed, among the references given in the chapter of Dickson's History devoted to irregular determinants, [Dickson 1923, pp. 89-91], Mordell is the only one to use this word "index" to refer to the same notion. ${ }^{61}$

In one of these cases, he supposed $k<0$ and congruent to 2 or 3 modulo 4 , and that the index of irregularity of $k$ is not divisible by 3 . Under these conditions he proved that if $(p, q)$ satisfies $q^{2}-k=p^{3}$, and $k$ is not of the form $-3 a^{2} \pm 1$, the classes of cubic forms of order 1 or 3 are those of the principal form and of $(1,0,-p, \pm 2 q)$. From this, he deduced that all the solutions of $y^{2}-k f^{2}=x^{3}$ are given by $x=p m^{2}-2 q m n+p^{2} n^{2}$, where $m, n$ are two integers such that $(1,0,-p, 2 q)(m, n)=f .{ }^{62}$

As for the last case, the same hypothesis was made on the index of irregularity, but $k$ was supposed to be equal to $-8 n-3$ for some positive integer $n$. These conditions, Mordell asserted, imply that the only (classes of) quadratic forms of order 1 or 3 are $(2 n+1, \pm 1,4)$ and $(1,0,-k)$, and these forms are the Hessians of the cubics $(n, \mp 1,-2,0)$ and $(0,1,0, k)$ : this result, which is not in Arndt's 1857 paper, appears in [Mathews 1891, p. 74]. As there is only a finite number of representations of $f$ by them-just as before, this is caused by the absence of terms $y^{3}$ or $x^{3}$ in these cubics-this proved that the equation $y^{2}-k f^{2}=x^{3}$ has a finite number of solutions.

Still, in this case Mordell even managed to show that, under the supplementary hypotheses that $f=1$ and that $k$ is of the form $-3 a^{2} \pm 1$ (resp. $-3 a^{2} \pm 8$ ), the only solution was given by $x=4 a^{2} \pm 1$ (resp. $a^{2} \pm 2$ ). At this occasion he cited his own note [Mordell 1913], as an "application to irregular determinants." In this note, Mordell used the previous result as follows: any integer $-k$ defined by $-k=x^{3}-y^{2}$, where $x$ and $y$ are integers (with $x$ not of the form $4 a^{2} \pm 1$ or $a^{2} \pm 2$ ) such that $x^{3}-y^{2}$ is negative, square-free, and congruent to 3 modulo 8 , is an irregular determinant whose index of irregularity is divisible by 3 . For instance, $x=11$ and $y=28$ were adequate values: with them, Mordell found that -547 is an irregular determinant. This allowed him to correct a table of Gauss [1876, pp. 417-476]

\footnotetext{
${ }^{61}$ Gauss had proved that classes of quadratic forms of given determinant $k$ can be grouped into genera, according to certain criteria. In modern parlance, if the genus $G_{0}$ containing the principal class is cyclic, the determinant $k$ is said to be regular; if not, it is irregular, and the quotient of the cardinality of $G_{0}$ by the maximal order of an element of $G_{0}$ is called the exponent (or the index, for Mordell) of irregularity of $k$. Let us additionally note that Mordell seemed to implicitly define the index of irregularity to be equal to 1 in the case of a regular determinant.

${ }^{62}$ We will see in our final section that this specific cubic form $(1,0,-p, 2 q)$ would be used by Alan Baker in subsequent works on the equation $y^{2}-k=x^{3}$.
} 
where -547 was counted as a regular determinant.

The three last paragraphs of the 1914 paper were devoted to explain how it was possible to find yet other supplementary hypotheses which would make the equation $y^{2}-k f^{2}=x^{3}$ impossible to solve in integers. These supplementary hypotheses, Mordell explained, served to bypass the lack of knowledge on the representations of integers by cubic forms: "At present, there is no general method of determining whether or not a given number can be represented by a given binary cubic; but, as before, we can obtain interesting results by considering congruences to various moduli." [Mordell 1914a, p. 77]. For instance, he showed that if $k f^{2} \equiv 4 \bmod 9$, then $f$ has no representation by any cubic form of determinant $4 k$, for the syzygy would lead to incompatible congruences. Thus, he concluded that the equation $y^{2}-k f^{2}=x^{3}$ has no solution if $k f^{2} \equiv 4 \bmod 9.63$

Contrary to the parts using the quadratic reciprocity law and ideal numbers, Mordell did not provide here any numerical instances of parameters $k$ and $f$ yielding equations with none or a finite number of solutions. However, the final paragraph of the paper proposed to "draw up a scheme, giving the values of $k$ between \pm 100 for which $y^{2}-k=x^{3}$ is soluble or not." [Mordell 1914a, p. 80]. Thus Mordell gave a list of 28 negative $k$ 's for which he believed that the corresponding equation had an infinite number of solutions-as remarked above, this is untrue because of Thue's theorem. The other values between -100 and -1 corresponded to none or a finite number of solutions, except for -31 and -84 , for which he admitted that "whether or not the equations are insoluble I cannot say." Similarly, Mordell listed 46 positive parameters $k$ yielding a finite (possibly null) number of solutions, declared that the others between 1 and 100 gave infinitely many solutions, except for 74 , about which he could not provide any conclusion.

Mordell did not provide any detail about these numerical values, such as from which part(s) of the paper they came. Among the parameters $k$ corresponding to a finite (or null) number of solutions and given in the final lists, we counted 21 of them that do not appear in the parts on congruences and on ideals: hence it is likely that they correspond to numerical examples found from the theory of cubic forms. For instance, $k=-59$ corresponds to the case where $k=-8 n-3$ and the corresponding index of irregularity if not divisible by 3 (as explained above Mordell knew tables listing class numbers and displaying the (ir)regularity of determinants).

\subsection{A glimpse at two futures of Mordell's paper: the survival of cubic forms}

As we wrote in our introduction, Mordell became Sadleirian professor at Cambridge in 1945 and he devoted his inaugural lecture to the equation $y^{2}-k=x^{3}$, a lecture that was published in 1947 under the title A Chapter in the Theory of Numbers, [Mordell 1947a]. In the lecture, after emphasizing the importance of this equation (together with the Pell equation $x^{2}-D y^{2}=1$ and the Fermat equation $x^{n}+y^{n}=z^{n}$ ) for the development of the theory of numbers, he rapidly expounded the research that had been done on it by some of his predecessors (such as Fermat, Lebesgue, Pépin, etc.). Mordell also summed up his own works on $y^{2}-k=x^{3}$ published in 1914, which he immediately connected to the 1922 finite basis theorem: "My continued interest and further work in this subject led me some ten years

${ }^{63}$ The conditions such as $k f^{2} \equiv 4 \bmod 9$ are reminiscent of those obtained in the part on ideal numbers, but Mordell did not comment on this. 
later to the proof of a fundamental theorem on the rational points of a cubic curve" [Mordell 1947a, p. 60]. In fact, the Chapter made clear that it was the part on cubic forms that had led Mordell to the 1922 theorem, through a process of successive (attempts at) generalizing works. Indeed, already in 1914 he published a paper where he showed (with the help of a syzygy) how to associate the integral solutions of $E y^{2}=A x^{3}+B x^{2}+C x+D$ to the representation of given numbers by given binary quartic forms, [Mordell 1914b]. Then, after having learned of Thue's theorem, he came back on the same equation in papers where he proved that it always has a finite number of integral solutions, [Mordell 1922a, 1923]. Finally, as he was trying to extend this result to equations of the form $y^{2}=A x^{4}+B x^{3}+C x^{2}+D x+E$, he arrived at the ideas that would lead to the finite basis theorem, [Mordell 1922b].

Through this narrative whose vanishing point was the finite basis theorem, Mordell thus emphasized the importance of the specific part of the 1914 paper on $y^{2}-k=x^{3}$ dealing with cubic forms-accordingly, this is essentially how this paper appears in the existing historiography devoted of the finite basis theorem, [Cassels 1973, 1986; Goldstein 1993]. But it is remarkable that the approach via the cubic forms also appears as central when one follows another path, that of the works of other mathematicians who took interest in the integral solutions of $y^{2}-k=x^{3}$.

Such works can be collected from a 1969 book by Mordell entitled Diophantine Equations, [Mordell 1969], which included a chapter on the equation $y^{2}-k=x^{3}$ together with ten references dealing with the integral solutions. Most of these works aimed at explicitly solving (some of) the equations $y^{2}-k=x^{3}$ with $|k| \leq 100$, such as [Nagell 1930; Hemer 1954; Ljunggren 1963], while others computed the solutions of the equation with $0 \leq x<10^{10}$ for all $|k|<9999$, [Lal, Jones, and Blundon 1966], or proved that the solutions satisfy $\max (|x|,|y|)<\exp \left(10^{10}|k|^{10^{4}}\right)$ for any non-zero parameter $k$, [Baker 1968]. ${ }^{64}$ Now, except for Alan Baker's paper, none of these references cited Mordell's 1914 article or even made allusions to it-when a work of Mordell is cited in them, it is mainly the Chapter in the Theory of Numbers, given as a historic and bibliographic reference. Yet they all stated in their introduction that solving $y^{2}-k=x^{3}$ in integers was equivalent to solving a finite number of equations of the form $f(u, v)=m$, where $f$ is a binary cubic. ${ }^{65}$ This technical result, which was in the 1914 paper of Mordell, was thus taken as a well-known starting point for further research. As for Baker, who cited this paper, he explained that his proof for establishing the boundary $\max (|x|,|y|)<\exp \left(10^{10}|k|^{10^{4}}\right)$ used techniques of Mordell, namely the introduction of the specific cubic form $(1,0,-p, 2 q)$, where $p, q$ is a given solution of $y^{2}-k=x^{3} .66$

All this provides a certain perspective on the future of the paper, and, if this perspective and that placing this paper in the shadow of the finite basis theorem are different, they do have in common retaining the specific part linking the solutions of $y^{2}-k=x^{3}$ with the

\footnotetext{
${ }^{64}$ The only reference whose mathematical objective does not fit in this trichotomy is the paper [Hall 1953], in which Marshall Hall aimed at finding specific conditions on $k$ giving insoluble equations.

${ }^{65}$ See for instance [Hemer 1954, p. 67] or [Lal, Jones, and Blundon 1966, pp. 322-323].

${ }^{66}$ See [Baker 1968, pp. 194-196]. More precisely, Baker first established a new proof of Thue's theorem yielding a boundary for the solutions of equations $f(x, y)=m$, where $f$ is an irreducible binary form, and combined the techniques of [Mordell 1914a] with the theory of reduction of cubic forms as grounded by Hermite and modified later by Davenport. As C. Goldstein pointed out to us, these works are part of those for which he was awarded the Fields Medal in 1970.
} 
theory of cubic forms. ${ }^{67}$

\section{A mathematical text and its asperities}

Throughout our description of the 1914 paper of Mordell, we have identified and brought to light a number of characteristics of this text which we interpreted as problematic features, as asperities of the text, and which generated a number of subsequent difficulties for its understanding. Thus, apart from the unstable notations, the undefined personal terminology, and the missing computations, arguments and explanations, we saw that the Mordell's way of writing produced overly allusive propositions having an unclear status (as well-known results, propositions to be proved, or surreptitiously introduced new hypotheses), and that the imprecision of a few technical passages made them approach the mathematical mistake.

Further, comprehension of the elliptic proofs was not made easier to us by the way Mordell referred to the works of other mathematicians, since in most of the cases where he explicitly cited such a work, he did not specify what he borrowed from it. For instance, the research of Fermat, Lebesgue, Gerono, Jonquières, Realis, and Pépin were seemingly cited at the very beginning of the paper as references of historic or bibliographic nature, and even if we detected a few resemblances between them and the method and results of the first part of Mordell's article, they did not help us fill the gaps in the corresponding proofs. Moreover, and although Mordell claimed the opposite, we noted that the cited article of Arndt was not sufficient to cover everything that was needed to understand the solution of $y^{2}-k=x^{3}$ with the help of cubic forms. ${ }^{68}$

As we aimed at completing Mordell's arguments and at accessing his possible sources, we also tried at several places to examine the vocabulary, the notation, and the results that he could have tacitly borrowed from other works. In the case of cubic forms, for example, we interpreted the use of the word "Hessian" as a hint to hidden English sources of the subject, sources from which we managed to understand Mordell's proof. Quite similarly, we remarked that the reciprocity law, the results on divisors of numbers of the form $x^{2}-k y^{2}$, and those on quadratic forms were all contained in Mathews' Theory of Numbers, which thus appeared as a probable source for Mordell. ${ }^{69}$ Nevertheless, we also showed that it was far from obvious how to reconstitute the sources of Mordell used for his ideal numbers (about which nothing was cited), as neither the appellation of these objects nor the way Mordell manipulated them allowed us to situate him with certainty in any of the known tradition on ideals or ideal numbers.

\footnotetext{
${ }^{67}$ Yet another perspective would be given by looking at works dealing with the rational solutions of the equation. In the works bearing on this problem and which are listed in [Mordell 1969], the 1914 paper appears neither explicitly, nor implicitly; in particular, the theory of cubic forms as it appeared in this paper is completely absent. The basic tool of the works on the rational problem seems to be the use of a factorization in an algebraic number field, but we do not think that this is to be specifically linked with the 1914 approach of Mordell.

${ }^{68}$ As we said earlier, this appreciation may just reflect that Mordell considered the knowledge on cubic forms that was not in Arndt's paper as elementary or common knowledge, and other readers could agree with Mordell that "all [that is needed] is contained" in the article of Arndt.

${ }^{69}$ The listed elements may be too common at the beginning of the twentieth century to serve as a proof that Mathew's book was a source for Mordell, but we recall that the latter cited this book in other papers published in the 1910s, and that he retrospectively listed Mathews as one of the rare number theorist in Great Britain at the time.
} 
As we noted at several places above, the asperities of Mordell's text can be differentiated according to the impact of the difficulties they generate, difficulties that depend on the reader. Thus for a specialist of number theory used to read papers with numerous omissions, it is probable that the sources to be used are obvious, that many of the blanks can be easily completed, and that the succession of Mordell's arguments is crystal clear; moreover, as unpleasant they can be, the instability of the notation and the uncertainty of the status of some statements certainly do not lead to major problems. But the reactions of some of Mordell's contemporary readers (which we reported in our section 1.3) prove that some of them did see his writing as problematic. In our opinion, this could reflect the fact that, as benign as they could be when taken one by one, the imprecise elements were accumulated to a too great extent, but also that they could lead to question the validity of some proofs in some cases.

Whatever be the difficulties and the questions they raise for the historian, we think it is important to take into consideration all these textual asperities in a historic study of Mordell's article, for they are direct traces of some aspects of his youthful mathematical practice. In other words, we think that the form of the writing, the way Mordell either expresses arguments and statements, or skips computations and bibliographic references, are elements which are historically meaningful, just as the results, the objects, the proofs, and the techniques themselves. In particular, taking all this into consideration allows us to get a finer understanding of the descriptions (including his own) which presented Mordell as a self-taught mathematician. Indeed, the close reading of his paper showed that he knew and manipulated with ease a great number of mathematical results, techniques, and sources. Hence Mordell's arithmetical self-education is less to be linked to a lack of mathematical resources than to a poor knowledge of the codes and the rules for writing a paper meant to be read by others: this was a crucial deficiency in Mordell's mathematical training, which seems to have had a genuine impact on the reception of some of his works. These remarks thus concretize (to a certain extent at least) the image of the self-taught Mordell in a way different from one that focused on the lectures he took or the books he read on his own, for instance.

Our choice to emphasise what we have called the asperities of Mordell's text particularly brings to the fore a writing "marred by obscurities of style," as a referee could have said (see p. 8) and his very personal way of manipulating mathematical objects. These features, together with the fact that almost all of the references given by Mordell in his paper were written by continental mathematicians and had been all published at least twenty years before 1914, tend to reinforce the image of the isolated self-taught number theorist that Mordell depicted of himself years later.

But our analysis of the 1914 paper also displays other facets of Mordell and his research. Our description of the results and the proofs sheds light on the ability of Mordell to grasp three distinct theories for tackling the equation $y^{2}-k=x^{3}$ and to carry out lengthy and technical computations resulting in many new results of solubility or insolubility of this equation. On the other hand, we saw how the explicit citations of Mordell's text brings it closer to the works on Diophantine equations published in Nouvelles annales de mathématiques at the end of the nineteenth century, and thus emphasizes the first part of the text and tends to obliterate (partially or completely) the other ones, where almost no papers were cited. ${ }^{70}$ Finally, the

\footnotetext{
${ }^{70}$ Situating a text into (a) network(s) of texts has been the object of some recent research in the history of mathematics. See for instance [Goldstein and Schappacher 2007b; Brechenmacher 2007].
} 
examples of the two future series of readings of Mordell's article ${ }^{71}$ that we examined at the end of our explanation of the part dealing with cubic forms give priority to this part, being the one which has been subsequently retained by people interested in the finite basis theorem or in further research on the integral solutions of $y^{2}-k=x^{3}$.

Such different focuses thus make different images of Mordell and his paper emerge, but these images are not incompatible. ${ }^{72}$ They complete each other and, together, yield a rich picture of the situation, displaying the tools that the young Mordell had at hand, and the way he used them to tackle a Diophantine equation that he was to follow throughout his whole career.

\section{Acknowledgements}

We would like to thank Jeremy Gray for his valuable suggestions which allowed us improve the paper, as well as for his precious linguistic help. We are also grateful to Catherine Goldstein and Norbert Schappacher for the discussions they had with us about the content of this paper.

\section{References}

Arndt, Friedrich (1857), "Zur Theorie der binären kubischen Formen”, Journal für die reine und angewandte Mathematik 53, pp. 309-321.

Baker, Alan (1968), "The Diophantine Equation $y^{2}=x^{3}+k$ ", Philosophical Transactions of the Royal Society of London. Series A, Mathematical and Physical Sciences 263, pp. 193-208.

Barrow-Green, June (1999), ““A Corrective to the Spirit of too Exclusively Pure Mathematics”: Robert Smith (1689-1768) and his Prizes at Cambridge University", Annals of Science 56 (3), pp. 271-316.

- (2014), "Cambridge Mathematicians' Responses to the First World War", in David Aubin and Catherine Goldstein (eds.), The War of Guns and Mathematics. Mathematical Practices and Communities in France and its Western Allies around World War I, Providence: American Mathematical Society, pp. 59-124.

Barrow-Green, June and Gray, Jeremy (2006), "Geometry at Cambridge, 1863-1940", Historia Mathematica 33, pp. 315-356.

Boniface, Jacqueline (2004), Hilbert et la notion d'existence en mathématiques, Paris: Vrin. Boucard, Jenny (2013), "Cyclotomie et formes quadratiques dans l'œuvre arithmétique d'Augustin-Louis Cauchy (1829-1840)", Archive for History of Exact Sciences 67 (4), pp. 349-414.

- (2019), "A Case Study About Mathematical Journals for Teachers and Students: Number Theory in the Nouvelles annales de mathématiques (1842-1927)", to appear in Revue d'histoire des mathématiques.

\footnotetext{
${ }^{71}$ On the question of the different interpretations of a text or a theorem by different readers, see [Goldstein 1995; Ehrhardt 2012].

${ }^{72} \mathrm{On}$ the effect of the construction of various contexts of reading, see [Ritter 2004].
} 
Brechenmacher, Frédéric (2007), "L'identité algébrique d'une pratique portée par la discussion sur l'équation à l'aide de laquelle on détermine les inégalités séculaires des planètes (1766-1874)", Sciences et techniques en perspective, 2nd ser. 1, pp. 5-85.

Cassels, John William Scott (1973), "Louis Joel Mordell 1888-1972", Biographical Memoirs of Fellows of the Royal Society 19, pp. 493-520.

- (1974), "L. J. Mordell”, Bulletin of the London Mathematical Society 6, pp. 69-96.

- (1986), "Mordell's Finite Basis Theorem Revisited", Mathematical Proceedings of the Cambridge Philosophical Society 100, pp. 31-41.

Cayley, Arthur (1857), "Note sur l'équation $x^{2}-D y^{2}= \pm 4, D \equiv 5(\bmod 8)$ ", Journal für die reine und angewandte Mathematik 53, pp. 369-371.

- (1858), "A Fifth Memoir upon Quantics", Philosophical Transactions of the Royal Society of London 148, pp. 429-460.

Cayley, Arthur and Hermite, Charles (1857), “On Cubic Forms”, The Quarterly Journal of Pure and Applied Mathematics 1, pp. 85-91.

Crilly, Tony (1988), "The Decline of Cayley's Invariant Theory (1863-1895)", Historia Mathematica 15 (4), pp. 332-347.

- (2006), Arthur Cayley: Mathematician Laureate of the Victorian Age, Baltimore: The John Hopkins University Press.

- (2011), "Cambridge. The Rise and Fall of the Mathematical Tripos", in Raymond Flood, Adrian C. Rice, and Robin J. Wilson (eds.), Mathematics in Victorian Britain, New York: Oxford University Press, pp. 17-32.

Cunningham, Allan (1905), "Solution to question 15697. Find all the integral solutions, if possible, of the equation $x^{2}-17=y^{3}$ ", Mathematical Questions and Solutions from "The Educational Times", 2nd ser. 8, pp. 53-54.

- (1908), "Solution to question 16408", Mathematical Questions and Solutions from "The Educational Times", 2nd ser. 14, pp. 106-108.

Davenport, Harold (1964), “L. J. Mordell”, Acta Arithmetica 9, pp. 3-12.

Despeaux, Sloan E. (2014), "Mathematical Questions: A Convergence of Mathematical Practices in British Journals of the Eighteenth and Nineteenth Centuries", Revue d'histoire des mathématiques 20, pp. 5-71.

Dickson, Leonard Eugene (1920), History of the Theory of Numbers, vol. 2. Diophantine Analysis, Washington: The Carnegie Institution of Washington.

- (1923), History of the Theory of Numbers, vol. 3. Quadratic and Higher Forms, Washington: The Carnegie Institution of Washington.

Duverney, Daniel (2010), Number Theory. An Elementary Introduction Through Diophantine Problems, Singapore: World Scientific.

Edwards, Harold M. (1980), "The Genesis of Ideal Theory", Archive for History of Exact Sciences 23, pp. 322-378.

- (1983), "Euler and Quadratic Reciprocity", Mathematics Magazine 56 (5), pp. 285-291.

Ehrhardt, Caroline (2012), Itinéraires d'un texte mathématique : les réélaborations des écrits d'Évariste Galois au XIX ${ }^{e}$ siècle, Paris: Hermann.

Euler, Leonhard (1822), Elements of Algebra, 3rd ed., London: Longman, Rees, Orme, and co.

Fairbairn, Ben (2017), “Louis Joel Mordell's Time in London”, BSHM Bulletin: Journal of the British Society for the History of Mathematics 32 (2), pp. 160-169. 
Ficklin, Joseph (1875), The Complete Algebra, New York, Chicago: Ivison, Blakeman, Taylor \& Co.

Fisher, Charles S. (1966), "The Death of a Mathematical Theory: A Study in the Sociology of Knowledge", Archive for History of Exact Sciences 3 (2), pp. 137-159.

Fletcher, Colin R. (1986), "Refugee Mathematicians: A German Crisis and a British Response, 1933-1936", Historia Mathematica 13, pp. 13-27.

Gauss, Carl Friedrich (1876), Carl Friedrich Gauss Werke, vol. 2, Göttingen: Königlichen Gesellschaft der Wissenschaften.

Gauthier, Sébastien (2007), "La géométrie des nombres comme discipline (1890-1945)", $\mathrm{PhD}$ thesis, Paris: Université Pierre et Marie Curie.

- (2009), "La géométrie dans la géométrie des nombres : histoire de discipline ou histoire de pratiques à partir des exemples de Minkowski, Mordell et Davenport", Revue d'histoire des mathématiques 15, pp. 183-230.

Gerono, Camille (1877), "Sur l'impossibilité de résoudre en nombres entiers l'équation $x^{3}=y^{2}+17$ ", Nouvelles annales de mathématiques, 2nd ser. 16, pp. 325-326.

Goldstein, Catherine (1993), "Descente infinie et analyse diophantienne : programmes de travail et mise en œuvre chez Fermat, Levi, Mordell et Weil", Cahiers du Séminaire d'histoire et de philosophie des mathématiques, 2nd ser. 3, pp. 25-49.

- (1995), Un théorème de Fermat et ses lecteurs, Saint-Denis: Presses Universitaires de Vincennes.

- (1999), "Sur la question des méthodes quantitatives en histoire des mathématiques : le cas de la théorie des nombres en France (1870-1914)", Acta historiae rerum naturalium necnon technicarum 3, pp. 187-214.

- (2009), "L'arithmétique de Fermat dans le contexte de la correspondance de Mersenne : une approche micro-sociale", Annales de la faculté des sciences de Toulouse 18, pp. 2557.

- (2015), "Axel Thue in Context", Journal de théorie des nombres de Bordeaux 27, pp. 309-337.

Goldstein, Catherine and Schappacher, Norbert (2007a), "A Book in Search of a Discipline”, in Catherine Goldstein, Norbert Schappacher, and Joachim Schwermer (eds.), The Shaping of Arithmetic after C. F. Gauss's Disquisitiones Arithmeticae, Berlin: Springer, pp. 3-65.

- (2007b), "Several Disciplines and a Book", in Catherine Goldstein, Norbert Schappacher, and Joachim Schwermer (eds.), The Shaping of Arithmetic after C. F. Gauss's Disquisitiones Arithmeticae, Berlin: Springer, pp. 67-103.

Grattan-Guinness, Ivor (1993), "The Sylvester Medal: Origins, and Recipients 1901-1949”, Notes and Records of the Royal Society of London 47 (1), pp. 105-108.

Hackley, Charles W. (1849), A Treatise on Algebra, 3rd ed., New York: Harper \& Brothers.

Hall, Marshall (1953), "Some Equations $y^{2}=x^{3}-k$ without Integer Solutions", Journal of the London Mathematical Society 28, pp. 379-383.

Hemer, Ove (1954), "Notes on the Diophantine Equation $y^{2}-k=x^{3}$ ", Arkiv för Matematik 3, pp. 67-77.

Houzel, Christian (2004), "Poincaré et l'analyse diophantienne", in Régis Morelon and Ahmad Hasnaoui (eds.), De Zénon d'Elée à Poincaré : recueil d'études en hommage à Roshdi Rashed, Louvain, Paris: Peeters Leuven, pp. 221-236. 
Jonquières, Ernest de (1878a), "Détermination de certains cas généraux où l'équation $x^{3} \pm a=y^{2}$ n'admet pas de solution en nombres entiers", Nouvelles annales de mathématiques, 2nd ser. 17, pp. 374-380.

- (1878b), "Au sujet des cas d'impossibilité d'une solution en nombres entiers de l'équation $x^{3} \pm a=y^{2} "$, Nouvelles annales de mathématiques, 2nd ser. 17, pp. 514-515.

Lal, M., Jones, M. F., and Blundon, W. J. (1966), "Numerical Solutions of the Diophantine Equation $y^{3}-x^{2}=k "$, Mathematics of Computation 20, pp. 322-325.

Lebesgue, Victor-Amédée (1869), "Note sur quelques équations indéterminées", Nouvelles annales de mathématiques, 2nd ser. 8, pp. 452-456.

Legendre, Adrien-Marie (1830), Théorie des nombres, 3rd ed., vol. 1, Paris: Firmin-Didot.

Lejeune-Dirichlet, Johann Peter Gustav (1828), "Mémoire sur l'impossibilité de quelques équations indéterminées du cinquième degré", Journal für die reine und angewandte Mathematik 3, pp. 354-375.

Ljunggren, Wilhelm (1963), "On the Diophantine Equation $y^{2}-k=x^{3}$ ", Acta arithmetica 8 , pp. 451-463.

Mathews, George Ballard (1911), "Relations between Arithmetical Binary Cubic Forms and their Hessians", Proceedings of the London Mathematical Society, 2nd ser. 9, pp. 200204.

- (1891), "Irregular Determinants and Subtriplicate Forms", The Messenger of Mathematics 20, pp. 70-74.

- (1892), Theory of Numbers, Cambridge: Deighton, Bell and Co.

- (1893a), "On the Complex Integers Connected with the Equation $\theta^{3}-2=0$ ", Proceedings of the London Mathematical Society 24, pp. 319-327.

- (1893b), "On the Algebraical Integers Derived from an Irreducible Cubic Equation", Proceedings of the London Mathematical Society 24, pp. 327-336.

Meyer, Wilhelm Franz (1899), "Invariantentheorie", in Encyklopädie der mathematischen Wissenschaften mit Einschluss ihrer Anwendungen, vol. I. B. 2, Leipzig: Teubner, pp. 320403.

Mordell, Louis J. (1913), "Note on Irregular Determinants", Messenger of Mathematics 42, p. 124.

- (1914a), "The Diophantine Equation $y^{2}-k=x^{3}$ ", Proceedings of the London Mathematical Society, 2nd ser. 13, pp. 60-80.

- (1914b), "Indeterminate Equations of the Third and Fourth Degrees", The Quarterly Journal of Pure and Applied Mathematics 45, pp. 170-186.

- (1915), "Theta Functions in the Theory of the Modular Functions and Groups of Linear Substitutions Arising Therefrom", The Quarterly Journal of Pure and Applied Mathematics 46, pp. 97-124.

- (1918), "The Theory of Numbers", Science Progress 12, pp. 127-131.

- (1920), "A Statement by Fermat", Proceedings of the London Mathematical Society, 2nd ser. 18, pp. v-vi.

- (1922a), "Note on the Integer Solutions of the Equation $E y^{2}=A x^{3}+B x^{2}+C x+D$ ", Messenger of Mathematics 51, pp. 169-171.

- (1922b), "On the Rational Solutions of the Indeterminate Equations of the Third and Fourth Degrees", Proceedings of the Cambridge Philosophical Society 21, pp. 179-192. 
Mordell, Louis J. (1923), "On the Integer Solutions of the Equation $e y^{2}=a x^{3}+b x^{2}+c x+d$ ", Proceedings of the London Mathematical Society, 2nd ser. 21 (415-419).

- (1947a), A Chapter in the Theory of Numbers, Cambridge: Cambridge University Press.

- (1947b), "On some Diophantine Equations $y^{2}=x^{3}+k$ with no Rational Solutions", Archiv for Mathematik og Naturvidenskab 49, pp. 143-150.

- (1959), Reflections of a Mathematician, Cambridge: Cambridge University Press.

- (1966), "The Infinity of Rational Solutions of $y^{2}=x^{2}+k$ ", Journal of the London Mathematical Society 41, pp. 523-525.

- (1968), "On some Diophantine Equations $y^{2}=x^{3}+k$ with no Rational Solutions (II)", in Abhandlungen aus Zahlentheorie und Analysis. Zur Erinnerung an Edmund Landau, Berlin: VEB Deutscher Verlag der Wissenschaften, pp. 225-232.

- (1969), Diophantine Equations, London and New York: Academic Press.

- (1971), "Reminiscences of an Octogenarian Mathematician", The American Mathematical Monthly 78, pp. 952-961.

Nagell, Trygve (1930), "Einige Gleichungen von der Form $a y^{2}+b y+c=d x^{3}$ ", Avhandlinger utgitt av det Norske Videnskaps-Akademi i Oslo. I: Matematisk-naturvidenskapelig Klasse 7, pp. 3-15.

Parshall Hunger, Karen (1989), "Toward a History of Nineteenth-Century Invariant Theory", in John McCleary and David E. Rowe (eds.), The History of Modern Mathematics, vol. 1. Ideas and their Reception, Boston, San Diego, New York: Academic Press, pp. 157-206.

Pépin, Théophile (1875), "Sur certains nombres complexes compris dans la formule $a+b \sqrt{-c}$ ", Journal de Mathématiques pures et appliquées, 3rd ser. 1, pp. 317-372.

- (1882), "Sur le problème de former un carré en ajoutant un cube à un nombre donné", Annales de la société scientifique de Bruxelles 6, pp. 86-100.

Prost, Antoine (1996), Douze leçons sur l'histoire, Paris: Éditions du Seuil.

Réalis, Savino (1883), "Sur une équation indéterminée", Nouvelles annales de mathématiques, 3rd ser. 2, pp. 289-297.

Rice, Adrian C. and Wilson, Robin J. (2003), "The Rise of British Analysis in the Early 20th Century: The Role of G.H. Hardy and the London Mathematical Society", Historia Mathematica 30, pp. 173-194.

Ritter, Jim (2004), "Reading Strasbourg 368: A Thrice-Told Tale", in Karine Chemla (ed.), History of Science, History of Text, Dordrecht: Springer, pp. 177-200.

Rollet, Laurent and Nabonnand, Philippe (2013), "Un journal pour les mathématiques spéciales : les Nouvelles annales de mathématiques (1842-1927)", Bulletin de l'Union des Professeurs de Spéciale 86, pp. 5-18.

Roquette, Peter (1998), “Zur Geschichte der Zahlentheorie in den dreißiger Jahren”, Mathematische Semesterberichte 45 (1), pp. 1-38.

Schappacher, Norbert (1991), "Développement de la loi de groupe sur une cubique", in Séminaire de théorie des nombres de Paris 1988/89, Catherine Goldstein (ed.), vol. 91, Basel, Boston, Berlin: Birkhäuser, pp. 159-184.

— (1998), "Eisenstein", in Heinrich Begehr, Jürg Kramer, Helmut Koch, Norbert Schappacher, and Ernst-Jochen Thiele (eds.), Mathematics in Berlin, Basel, Boston, Berlin: Birkhäuser, pp. 55-60. 
Schappacher, Norbert (2005), "David Hilbert, Report on Algebraic Number Fields ('Zahlbericht') (1897)", in Ivor Grattan-Guinness (ed.), Landmark Writings in Western Mathematics 1640-1940, Amsterdam: Elsevier, chap. 54, pp. 700-709.

Schappacher, Norbert and Schoof, René (1996), "Beppo Levi and the Arithmetic of Elliptic Curves", The Mathematical Intelligencer 18 (1), pp. 57-69.

Smith, Henry John Stephen (1860), "Report on the Theory of Numbers, Part II", Report of the British Association for the Advancement of Science, pp. 120-169. Reproduced in The Collected Mathematical Papers, James Whitbread Lee Glaisher (ed.), Oxford: Clarendon Press, pp. 93-162, 1894.

Sommer, Julius (1907), Vorlesungen über Zahlentheorie: Einfuhrung in die Theorie der algebraischen Zahlkörper, Leipzig, Berlin: Teubner.

Thue, Axel (1909), "Über Annäherungswerte algebraischer Zahlen”, Journal für die reine und angewandte Mathematik 135, pp. 284-305.

Titchmarsch, Edward Charles (1949), "Godfrey Harold Hardy, 1877-1947”, Obituary Notices of Fellows of the Royal Society 6, pp. 446-461.

Todd, John Arthur (1958), “John Hilton Grace 1873-1958”, Biographical Memoirs of Fellows of the Royal Society 4, pp. 92-97.

Warwick, Andrew (2003), Masters of Theory. Cambridge and the Rise of Mathematical Physics, Chicago: The University of Chicago Press.

Wilson, David B. (1982), "Experimentalists among the Mathematicians: Physics in the Cambridge Natural Sciences Tripos, 1851-1900", Historical Studies in the Physical Sciences 12 (2), pp. 325-371. 